TI 2016-022/IV

Tinbergen Institute Discussion Paper
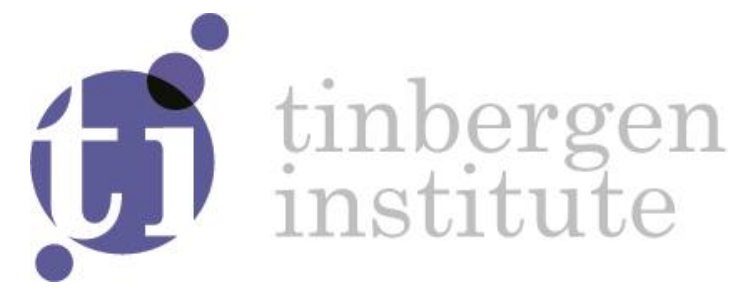

\title{
Single Stock Call Options as Lottery Tickets: Overpricing and Investor Sentiment
}

\section{REVISION: January 2018}

\author{
Luiz Félix ${ }^{1}$ \\ Roman Kräuss/ ${ }^{2}$
}

Philip Stork ${ }^{3}$

\footnotetext{
${ }^{1}$ APG Asset Management, VU University Amsterdam, The Netherlands

${ }^{2}$ Luxembourg School of Finance, University of Luxembourg, Luxemburg

${ }^{3}$ VU University; Tinbergen Institute, The Netherlands
} 
Tinbergen Institute is the graduate school and research institute in economics of Erasmus University Rotterdam, the University of Amsterdam and VU University Amsterdam.

Contact: discussionpapers@tinbergen.nl

More TI discussion papers can be downloaded at http://www.tinbergen.nl

Tinbergen Institute has two locations:

Tinbergen Institute Amsterdam

Gustav Mahlerplein 117

1082 MS Amsterdam

The Netherlands

Tel.: +31(0)205984580

Tinbergen Institute Rotterdam

Burg. Oudlaan 50

3062 PA Rotterdam

The Netherlands

Tel.: +31(0)10408 8900 


\title{
Single stock call options as lottery tickets: overpricing and investor sentiment*
}

\author{
Luiz Félix \\ VU University Amsterdam \\ APG Asset Management
}

\author{
Roman Kräussl \\ Luxembourg School of Finance, \\ University of Luxembourg
}

\author{
Philip Stork $^{\dagger}$ \\ VU University Amsterdam \\ Tinbergen Institute Amsterdam
}

January 2018

\begin{abstract}
This paper investigates whether the overpricing of out-of-the money single stock calls can be explained by Tversky and Kahneman's (1992) cumulative prospect theory (CPT). We hypothesize that these options are overpriced because investors overweight small probability events and overpay for positively skewed securities, i.e, lottery tickets. We find that overweighting of small probabilities embedded in the CPT explains the richness of out-of-the money single stock calls better than other utility functions. Nevertheless, overweighting of small probabilities events is less pronounced than suggested by the CPT, is strongly time-varying and most frequent in options of short maturity. Fluctuations in overweighting of small probabilities are largely explained by the sentiment factor.
\end{abstract}

JEL classification: G02, G12.

Keywords: Cumulative prospect theory; investor sentiment; risk-neutral densities; call options.

${ }^{*}$ We thank seminar participants at the IFABS 2016 Barcelona Conference, at the VU University Amsterdam, at the APG Asset Management Quant Roundtable, at the 2016 Research in Behavioral Finance Conference in Amsterdam and at the Board of Governors of the Federal Reserve System for their helpful comments. We thank APG Asset Management for financial support and for making available part of the data.

${ }^{\dagger}$ Philip Stork (corresponding author) is Professor at VU University Amsterdam, FEWEB, School of Finance and Risk Management, de Boelelaan 1105, 1081 HV Amsterdam, The Netherlands. Tel.: + 31205983651. E-mail address: p.a.stork@vu.nl. 


\section{Introduction}

Barberis and Huang (2008) hypothesize that Tversky and Kahneman's (1992) cumulative prospect theory (CPT) explains a number of seemingly unrelated pricing puzzles. In contrast to previous literature, which concentrates on the CPT's value function (see Benartzi and Thaler, 1995; Barberis et al., 2001; Barberis and Huang, 2001), Barberis and Huang (2008) focus on the probability weighting functions of the model. They conclude that the CPT's overweighting of small probability events explains why investors prefer positively skewed returns, or "lottery ticket" type of securities. Because of such preference, investors overpay for positively skewed securities, turning them expensive and causing them to yield low forward returns. The authors argue that this mechanism is the reason why IPO stocks, private equity, distressed stocks, single segment firms and deep out-of-the money (OTM) single stock calls are overpriced among other irrational pricing phenomena.

The proposition made by Barberis and Huang (2008) that deep OTM single stock calls resemble overpriced lottery-like securities due to investors' overweight of tails has not yet been verified empirically ${ }^{1}$. Empirical studies on probability weighting functions implied by option prices are offered by Dierkes (2009), Kliger and Levy (2009), and Polkovnichenko and Zhao $(2013)^{2}$. The evidence provided by these papers is, however, based on the index put options market, which behaves very differently from the single stock option market. The main buyers of OTM index puts are institutional investors, which use them for portfolio insurance (Bates, 2003; Bollen and Whaley, 2004; Lakonishok et al., 2007; Barberis and Huang, 2008). Because institutional investors comprise around two-thirds of the total equity market capitalization (Blume and Keim, 2012), their option trading activity strongly impacts the pricing of put options (Bollen and Whaley, 2004) by making them expensive. The results of Dierkes (2009) and Polkovnichenko and Zhao (2013) reiterate this evidence and suggest that overweighting of small probabilities partially explains the pricing puzzle present in the equity index option market.

Contrary to the index put market, trading activity in single stock calls is concentrated among individual investors (Bollen and Whaley, 2004; Lakonishok et al., 2007) and is speculative in nature (Lakonishok et al., 2007; Bauer et al., 2009; Choy, 2015). Beyond that, Mitton and Vorkink (2007); Bauer et al. (2009); Kumar (2009) provide important empirical support to the link between preference for skewness and individual investor trading activity. The fact that many individual investors have a substantial portion of their portfolios tied up in low risk investments, such as pensions, social security, 401(k)s, IRAs, or are averse (or constrained) to borrow (Frazzini and Pedersen, 2014) encourages them to buy financial instruments with

\footnotetext{
${ }^{1}$ Boyer and Vorkink (2014) provide evidence that lottery-like single stock options do deliver lower forward returns than options with lower ex-ante skewness. However, their paper does not test why these options are overvalued, nor does it analyse the potential time-variation in ex-ante skewness and forward returns. Conrad et al. (2013) find similar results for ex-ante skewness and subsequent stock returns.

${ }^{2}$ These studies focus on the rank-dependent expected utility (RDEU) rather than the CPT, as the RDEU is seamlessly effective in dealing with the overweighting of probability phenomena. The RDEU's probability weighting functions are strictly monotonically increasing, whereas the CPT one is not. RDEU functions are also easier to estimate because they use one less parameter than the CPT.
} 
implicit leverage such call options. Hence, given the very distinct clientele of these two option markets (institutional investors vs. retail investors) and the different motivation for trading (portfolio insurance vs. speculation), we reason that the OTM single stock calls overpricing is a puzzle in itself, requiring an independent empirical proof from the index option market.

The first contribution of our study is to investigate whether the CPT can empirically explain the claimed overpricing of OTM single stock call options. To that purpose, we empirically test whether tails of the CPT density function outperform the risk-neutral density and rational subjective probability density functions on matching tails of the distribution of realized returns. We find that our estimates for the CPT probability weighting function parameter $\gamma$ are qualitatively consistent with the ones predicated by Tversky and Kahneman (1992), particularly for short-term options. Our estimates do suggest that overweight of small probabilities is less pronounced than suggested by the CPT though. This analysis complements the results of Barberis and Huang (2008) and provides novel support to explain the overpricing of OTM single stock calls. Our empirical results extend the findings of Dierkes (2009), Kliger and Levy (2009), Polkovnichenko and Zhao (2013), because we show that investors' overweighting of small probabilities is not restricted to the pricing of index puts but also applies to single stock calls.

Secondly, we provide evidence that overweighting of small probabilities is strongly timevarying and connected to the Baker and Wurgler (2007) investor sentiment factor. These findings contrast the CPT model, where the probability weighting parameter for gains $(\gamma)$ is constant at 0.61 . In fact, our estimations suggest that the $\gamma$ parameter fluctuates widely around that level, sometimes even reflecting underweighting of small probabilities. We show that overweighting of small probabilities was quite strong during the dot-com bubble, which coincided with a strong rise in investor sentiment. The strong time-variation in overweight of tails indicates that investors have either a "bias in beliefs" or time-varying (rather than static) skewness preferences, see Barberis (2013) for a discussion on the topic ${ }^{3}$.

Moreover, we find that overweighting of small probabilities is largely horizon-dependent, because this bias is mostly observed within short-term options prices (i.e., three- and sixmonths) rather than in long-term ones (i.e., twelve-months). We reason that such positive term structure of tails' overweighting exist because individual investors may speculate using the cheapest available call at their disposal. In other words, individual investors buy the cheapest lottery tickets that they can find. As three- and six-month options have much less time-value than twelve-month ones, more pronounced overweighting of small probabilities within shortterm options seems sensible. This result is consistent with individual investors being the typical buyers of OTM single stock calls and the fact that they mostly use short-term instruments to speculate on the upside of equities (Lakonishok et al., 2007).

\footnotetext{
${ }^{3}$ We acknowledge that it is unclear whether overpicing of OTM calls is caused by overweighting of small probablities (i.e., a matter of preferences), or rather by biased beliefs. Barberis (2013) eloquently discusses how both phenomena are distinctly different and how both (individually or jointly) may explain the overpricing in OTM options. In this paper we take a myopic view and use only the first explanation, for ease of exposition. Disentangling the two (beliefs and preferences) would potentially be very interesting, but we deem it to be outside the scope of this paper.
} 
In our analysis of probability weighting functions, we focus on the outmost tails of RNDs ${ }^{4}$. We argue that, as distribution tails (mostly estimated from OTM options) are the sections of the distribution that reflect low probability events, we may analyze these locally, thus, isolated from the distribution's body. To this purpose, we use extreme value theory (EVT) and Kupiec's test (as a robustness check), which are especially suited for the analysis of tail probabilities and, so far, have not been employed yet to the evaluation of overpricing of OTM options. As an additional robustness check, we replace the CPT by the rank-dependent expected utility (RDEU) function of Prelec (1998). This alteration reconfirms the presence of overweighted small probabilities by investors within the OTM single stock call market and, at the same time, reiterates that such bias is less pronounced than suggested by the CPT model. Time-variation of the weighting function parameters is also observed when RDEU is applied.

The remainder of this paper is organized as follows. Section 2 describes the CPT model. Section 3 describes the data and methodology employed in our study. Section 4 presents our empirical analysis and Section 5 discusses our robustness tests. Section 6 concludes.

\section{Cumulative Prospect Theory}

The Prospect theory (PT) of Kahneman and Tversky (1979) incorporates behavioral biases into the standard utility theory (von Neumann and Morgenstern, 1947), which presumes that individuals are rational ${ }^{5}$. Such behavioral anomalies are i) loss aversion, ii) risk seeking behavior and iii) non-linear preferences ${ }^{6}$. The CPT is described in terms of a value function $(v)$ and a probability distortion function $(\pi)$. The value function is analogous to the utility function in the standard utility theory and it is defined relative to a reference point zero. Therefore, positive values within the value function are considered as gains and negative values are losses, which leads to:

$$
v(x)=\left\{\begin{array}{l}
x^{\alpha}, \text { if } x>=0 \\
-\lambda(-x)^{\beta}, \text { if } x<0
\end{array}\right.
$$

where $\lambda \geq 1,0 \leq \beta \leq 1,0 \leq \alpha \leq 1$, and $x$ are gains or losses. Thus, along the dominium

\footnotetext{
${ }^{4}$ Per contrast, Dierkes (2009) and Polkovnichenko and Zhao (2013) explore the relation between overweighting of small probabilities and options prices by analyzing the full RND from options. Dierkes (2009) applies Berkowitz's tests, whereas Polkovnichenko and Zhao (2013) estimate an empirical weighting function via polynomial regressions.

${ }^{5}$ The expected utility theory of von Neumann and Morgenstern (1947) is the standard economics framework on decision making under risk. Their theory assumes that decision-makers behave as if they maximize the expected value of some function defined over the potential (probabilitistic) outcomes. Individuals are assumed to have stable and rational preferences; i.e., not influenced by the context or framing.

${ }^{6}$ Loss aversion is the property in which people are more sensitive to (or affected by) losses than gains. For details, see Kahneman and Tversky (1979), Tversky and Kahneman (1992) and Barberis and Huang (2001). Risk-seeking behavior happens when individuals are attracted by gambles with unfair prospects. In other words, the risk-seeking individual is the one that chooses for a gamble versus a sure thing even though the two outcomes have the same expected value. For details, see Kahneman and Tversky (1979). Non-linear preferences occur when preferences between risky prospects are not linear in the probabilities, thus, equally probable prospects are more heavily weighted by agents than others. For details, see Tversky and Kahneman (1992), Fox et al. (1996), Wu and Gonzalez (1996), Prelec (1998) and Hsu et al. (2009).
} 
of $x$, the CPT's value function is asymmetrically $S$-shaped (see Figure 1A) with diminishing sensitivity as $x \rightarrow \pm \infty$.

The value function is, thus, concave over gains and convex over losses, differently from the traditional utility function used by standard utility theory. Such a shape of the value function implies diminishing marginal values as gains or losses increase, which means that any additional unit of gain (loss) becomes less relevant when wealth increases (decreases). As $\alpha$ and $\beta$ increase, the effect of diminishing sensitivity decreases, and as $\lambda$ increases the degree of loss aversion increases. We also note in Figure 1A that the value function has a kink at the reference point, which implies loss aversion, as the function is steeper for losses than for gains.

[Please insert Figure 1 about here]

The use of a probability distortion function or decision weight function is the adjustment made to the PT to address nonlinear preferences. This function takes probabilities and weights them nonlinearly, so that the difference between probabilities at high percentiles, e.g., between 99 percent and 100 percent, has more impact on preferences than the difference between probabilities at small percentiles, e.g., between 10 percent and 11 percent. This is the main advance of the CPT over the original PT. The CPT applies probability distortions to the cumulative probabilities (i.e., the $\mathrm{CDF}$ ), whereas the $\mathrm{PT}$ applies them to individual probabilities (i.e., the PDF). The enhancement brought by this new formulation satisfies stochastic dominance conditions not achieved by the PT, which renders the CPT applicable to a wider number of experiments. The probability distortion functions suggested by Tversky and Kahneman (1992), respectively, for gains $\left(\pi_{n}^{+}\right)$and losses $\left(\pi_{-m}^{-}\right)$are:

$$
\begin{gathered}
\pi_{n}^{+}=w^{+}\left(p_{n}\right) \\
\pi_{i}^{+}=w^{+}\left(p_{i}+\ldots+p_{n}\right)-w^{+}\left(p_{i+1}+\ldots+p_{n}\right), \text { for } 0 \leq i \leq n-1 \\
\pi_{-m}^{-}=w^{-}\left(p_{-m}\right) \\
\pi_{i}^{-}=w^{-}\left(p_{-m}+\ldots+p_{i}\right)-w^{-}\left(p_{-m}+\ldots+p_{i-1}\right), \text { for } 1-m \leq i \leq 0
\end{gathered}
$$

where $p$ are objective probabilities of outcomes, which are ranked for gains from the reference point $i=0$ to $i=n$, the largest gain, and for losses from the largest loss $i=-m$ to $i=0$, the reference point. Further, $w^{+}$and $w^{-}$, the parametric form of the decision weighting functions, are given by:

$$
\begin{aligned}
& w^{+}(p)=\frac{p^{\gamma}}{\left(p^{\gamma}+(1-p)^{\gamma}\right)^{1 / \gamma}} \\
& w^{-}(p)=\frac{p^{\delta}}{\left(p^{\delta}+(1-p)^{\delta}\right)^{1 / \delta}}
\end{aligned}
$$

where parameters $\gamma$ and $\delta$ define the curvature of the weighting function for gains and losses, which leads the probability distortion functions to assume inverse $S$-shapes. Figure $1 \mathrm{~B}$ depicts how low probability events are overweighted at the cost of moderate and high probabilities 
within the CPT probability distortion functions. Tversky and Kahneman (1992) indicate that the weighting functions for gains are slightly more curved than for losses (i.e., $\gamma<\delta$ ). The parameters estimated by the authors for the CPT model, which are discussed in our empirical analysis below, are $\lambda=2.25 ; \beta=0.88 ; \alpha=0.88 ; \gamma=0.61 ; \delta=0.69$.

\section{$3 \quad$ Data and Methodology}

In this section, we first describe the theoretical background that allows us to relate empirical density functions (EDF), RND, and subjective density functions. This is a key step for testing the hypothesis that the CPT helps to explain overpricing of OTM options, because we build on the assumption that investors' subjective density estimates should correspond, on average ${ }^{7}$, to the distribution of realizations (see Bliss and Panigirtzoglou, 2004). Thus, testing whether the CPT's weighting function explains the overpricing of OTM options, ultimately, relates to how the subjective density function produced by CPT's preferences matches empirical returns. Because the representative agent is not observable, subjective density functions are not estimable like EDF and RND are. As such, we build on the following theory to derive subjective density functions from RNDs.

In our empirical exercise, we first derive subjective density functions for (a) the power and (b) exponential utility functions. Because the CPT model contains not only a utility function (the value function) but also a probability weighting scheme (the weighting function), we produce two density functions: (c) the hereafter called partial CPT density function (PCPT), where only the value function is taken into account, and (d) the CPT density function, where the value and the weighting functions are considered. Lastly, we also calibrate $\gamma$ to market data and are, then, able to compute (e) the estimated CPT density (ECPT). We provide details on estimation methods for our five subjective density functions, (a) to (e), in Section 3.1, and for the RND and EDF in Section 3.4.

Once all five subjective density functions are obtained, we distinguish four analyses in our empirical analysis section: 1) the estimation of long-term CPT value and weighting function parameters (from which we can produce the ECPT density) (Section 4.1); 2) EVT-based tests of consistency between tails of the EDF, the RND and our five subjective probability distributions (Section 4.2); 3) the estimation of time-varying $\gamma$ parameter (Section 4.3); and 4) a regression linking the CPT time-varying probability weighting parameter $(\gamma)$ to sentiment measures as well as numerous control variables (Section 4.4).

We use single stock weighted average IV data used for the largest 100 stocks of the S\&P 500 index within our RND estimations. Appendix A.2 shows how single stock weighted average IV are computed. Weights applied are the S\&P 500 index weights normalized by the sum of weights of stocks for which IVs are available. Following the S\&P 500 index methodology and the

\footnotetext{
${ }^{7}$ This implies that investors are somewhat rational. This assumption is not inconsistent with the CPT assumption that the representative agent is less than fully rational. The CPT suggests that investors are biased, not that decision makers are utterly irrational to the point that their subjective density forecast should not correspond, on average, to the realized return distribution.
} 
unavailability of IV information for every stock in all days in our sample, stocks weights in this basket change on a daily basis. The sum of weights is, on average, 58 percent of the total S\&P 500 index capitalization and it fluctuates between 46 and 65 percent. The IV data comes from closing mid-option prices from January 2, 1998 to March 19, 2013 for fixed maturities for five moneyness levels, i.e., 80, 90, 100, 110, and 120, at the three-, six- and twelve-month maturity. Continuously compounded stock market returns are calculated throughout our analysis from the basket of stocks weighted with the same daily-varying loadings used for aggregating the IV data. IV data and stock weights are kindly provided by Barclays ${ }^{8}$. Single stock returns are downloaded via Bloomberg.

We take the perspective of end-users of single stock OTM call options ${ }^{9}$. Hence, we assume that supply imbalances are minimal and do not impact implied volatilities. We think this assumption is reasonable because 1) option markets for the largest 100 U.S. stocks are liquid; 2) any un-hedged risk run by market makers can be easily hedged by purchasing the stock; and 3) unhedged risk by market makers is likely much smaller when supplying call options relative to put options. Market makers run little unhedged risk when supplying call options vis-à-vis supplying puts because stocks returns are negatively skewed, making gap and jump risk much lower on the upside than on the downside. Garleanu et al. (2009) have shown that this condition is different for the index option market, where market makers mostly provide put options for portfolio insurance programs. As the authors suggest, put sellers become more risk-sensitive following equity market declines, as their un-hedged risk increases, which makes them unwilling to write additional puts to the market. Our implied volatility data show no indication of an increase in the implied volatility skew from 120 percent moneyness options, nor from at-the-money options around moments of market stress (e.g., the 2008-09 global financial crisis). Hence, we find no evidence of the presence of supply imbalances in the OTM calls in our sample.

\subsection{Subjective density functions}

Standard utility theory tells us that since the representative agent does not have risk-neutral preferences, RNDs are inconsistent with subjective and $\mathrm{EDF}^{10}$, thus both "real-world" probabilities. Hence, if investors are risk-averse or risk seeking, their subjective probability function should differ from the one implied by option prices. The relation between the RND $f_{Q}\left(S_{T}\right)$,

\footnotetext{
${ }^{8}$ We thank Barclays for providing the implied volatility data. Barclays disclaimer: "Any analysis that utilizes any data of Barclays, including all opinions and/or hypotheses therein, is solely the opinion of the author and not of Barclays. Barclays has not sponsored, approved or otherwise been involved in the making or preparation of this Report, nor in any analysis or conclusions presented herein. Any use of any data of Barclays used herein is pursuant to a license."

${ }^{9}$ We implicitly assume that end-users of single stock OTM call options have the same preferences across underlyings. This assumption is supported by the evidence provided by Bollen and Whaley (2004) and Lakonishok et al. (2007) that trading activity in equity calls is concentrated among individual investors and is speculative in nature.

${ }^{10}$ Anagnou et al. (2002) and Bliss and Panigirtzoglou (2004) have tested the consistency between RNDs and physical densities estimated from historical data and found that such distributions are inconsistent, i.e., RNDs are poor forecasters of the distribution of realizations.
} 
and "real-world" probability distributions, $f_{P}\left(S_{T}\right)$, with $S_{T}$ being wealth or consumption"11, is described by $\varsigma\left(S_{T}\right)$, the pricing kernel or the marginal rate of substitution (of consumption at time $T$ for consumption at time $t)^{12}$ :

$$
\frac{f_{P}\left(S_{T}\right)}{f_{Q}\left(S_{T}\right)}=\Lambda \frac{U^{\prime}\left(S_{T}\right)}{U^{\prime}\left(S_{t}\right)} \equiv \varsigma\left(S_{T}\right),
$$

where $\Lambda$ is the subjective discount factor (the time-preference constant) $U(\cdot)$ is the representative investor utility function. As $U\left(S_{T}\right)$ is a random variable, the pricing kernel is also called the stochastic discount factor. Thus, Eq. (4) tell us that the "real-world" distribution equates to the RND when adjusted by the pricing kernel. The intuition behind Eq. (4) is that a real-world or risk-adjusted probability distribution can be obtained from the RND, once the risk trade-off embedded in the representative investor utility function is considered.

Since CPT-biased investors price options as if the data-generating process has a cumulative distribution $F_{\widetilde{P}}\left(S_{T}\right)=w\left(F_{P}\left(S_{T}\right)\right)^{13}$, where $w$ is the weighting function, its density function becomes $f_{\widetilde{P}}\left(S_{T}\right)=w^{\prime}\left(F_{P}\left(S_{T}\right)\right) \cdot f_{P}\left(S_{T}\right)$ (see Dierkes, 2009; Polkovnichenko and Zhao, 2013). Thus, CPT-biased agents assess probability distributions as if their tails would contain more weight than in reality they do, i.e., they have a preference for skewness or "bias in beliefs", as Barberis (2013) argues. Consequently, evaluating whether the CPT's propositions apply is equivalent to testing whether Eq. (4) still holds if $f_{P}\left(S_{T}\right)$ is replaced by $f_{\widetilde{P}}\left(S_{T}\right)$, leading to:

$$
\frac{w^{\prime}\left(F_{P}\left(S_{T}\right)\right) \cdot f_{P}\left(S_{T}\right)}{f_{Q}\left(S_{T}\right)}=\varsigma\left(S_{T}\right) .
$$

We, then, further manipulate Eq. (5) so to directly relate the original EDF to the CPT subjective density function, by "undoing" the effect of the CPT probability distortion functions within the PCPT density function. The relation between EDF and the CPT density function is given by Eq. (6) and its derivation, from Eq. (5), is provide in Appendix A.1:

$$
\underbrace{f_{P}\left(S_{T}\right)}_{\mathrm{EDF}}=\underbrace{\frac{\frac{f_{Q}\left(S_{T}\right)}{\nu^{\prime}\left(S_{T}\right)}}{\int \frac{f_{Q}(x)}{\nu^{\prime}(x)} d x}\left(w^{-1}\right)^{\prime}\left(F_{P}\left(S_{T}\right)\right)}_{\text {CPT density function }}
$$

where $\nu^{\prime}\left(S_{T}\right)$ is the CPT's marginal utility function.

This result allows us to obtain a clear representation of the CPT subjective density function, thus, where the value and the weighting function are simultaneously taken into account. At this stage, as we can produce RND and the set of subjective densities of our interest, including

\footnotetext{
${ }^{11}$ Note that, as the value function within the CPT measures utility versus a reference point, $S_{T}$ is not strictly positive in this model. A negative $S_{T}$ denotes a loss of wealth or consumption, whereas a positive $S_{T}$ represents a gain.

${ }^{12}$ The condition necessary for Eq. (4) to hold is that markets are complete and frictionless and a single risky asset is traded.

${ }^{13}$ Similarly, if investors are rational, their subjective density functions should be consistent, on average, with the empirical density function. Bliss and Panigirtzoglou (2004) find that subjective density functions, produced from RND adjusted by two types of representative investors' utility functions (power and exponential) with plausible relative risk aversion parameters, outperform RND on forecasting density functions.
} 
the CPT density, one can evaluate how consistent with realizations their tails are.

\subsection{Estimating CPT parameters}

We start evaluating the empirical validity of the CPT for single stock call options by comparing EDF to the CPT density function parameterized by Tversky and Kahneman (1992). Subsequently, we estimate CPT weighting function parameters $\lambda$ and $\gamma$ with the same goal. We only estimate $\gamma$ within the probability weighting function, and not $\delta$, because we are interested in the gains-side of the distribution, which is extracted from call options. We estimate these parameters non-parametrically, by minimizing the weighted squared distance between physical distribution and the partial CPT density function for every bin above the median of the two distributions, as follows:

$$
v(\lambda)=\operatorname{Min} \sum_{b=1}^{B} W_{b}\left(E D F_{p r o b}^{b}-C P T_{\text {prob }}^{b}\right)^{2},
$$

where, $E D F_{p r o b}^{b}$ and $C P T_{\text {prob }}^{b}$ are, respectively, the probability within bin $b$ in the empirical and CPT density functions and $W_{b}$ are weights given by $\frac{1}{\frac{1}{\sqrt{2}}} \int_{0.5}^{\infty} e^{\frac{-x^{2}}{2}} d x=1$, the reciprocal of the normalized normal probability distribution (above its median), split in the same total number of bins $(B)$ used for the EDF and CPT. The loss aversion parameter, $\lambda$, in Eq. (7) is optimized using multiple constraint intervals: $[0,3],[0,5]$ and $[0,10]$. Once the optimal $\lambda$ is known, we minimize Eq. (8) using its estimate and the $\operatorname{CPT} \lambda$ :

$$
w^{+}(\gamma, \delta=\gamma)=\operatorname{Min} \sum_{b=1}^{B} W_{b}\left(E D F_{p r o b}^{b}-C P T_{p r o b}^{b}\right)^{2},
$$

where $\gamma$, the probability weighting parameter for gains, is constrained by the permutation of the following upper bounds $(1.2,1.35,1.5,1.75$ and 2$)$ and lower bounds $(-0.25,0$ and 0.28). Weights applied in these optimizations are due to the higher importance of matching probabilities tails in our analysis than the body of the distributions.

Our non-linear bounded optimization is a single parameter one, where we first estimate optimal $\gamma$ (which we impose to equal $\delta$ ) across all permutations of upper and lower bounds to select the bounds that produce the lowest residual sum of square (RSS). Subsequently, we estimate $\lambda$ and $\gamma$ as suggested by the sequence of optimizations described by Eqs. (7) and (8). This method resembles the ones of Kliger and Levy (2009), Dierkes (2009), ChabiYo and Song (2013), and Polkovnichenko and Zhao (2013). Once optimal parameters $\lambda$ and $\gamma$ are estimated, we can produce another long-term subjective density function: the ECPT, which stands for estimated CPT, where we apply the optimal $\gamma$ for the characterization of its probability weighting function. Finally, we also estimate time-varying $\gamma$ using different assumptions of $\lambda$, so to evaluate the sensitivity of $\gamma$ to changes in $\lambda$. 


\subsection{Density function tails' consistency test}

We check for tail consistency of our set of five subjective density functions (CPT, PCPT, ECPT, power and exponential), RND, and the EDF by applying extreme value theory (EVT). EVT allows us to estimate the shape of the tails of these eight PDFs and to extract the returns implied by an extreme quantile within our PDFs. We estimate the tail shape estimator $(\varphi)$ by means of the Hill (1975) estimator:

$$
\hat{\varphi}=\frac{1}{\hat{\theta}}=\frac{1}{k} \sum_{j=1}^{K} \ln \left(\frac{x_{j}}{x_{k+1}}\right),
$$

where $k$ is the number of extreme returns used in the tail estimation, and $x_{k+1}$ is the tail cut-off point. The tail shape estimator $\varphi$ measures the curvature, i.e., the fatness of the tails of the return distribution: a high (low) $\varphi$ indicates that the tail is fat (thin). The inverse of $\varphi$ is the tail index $(\theta)$, which determine the tail probability's rate of decay. A high (low) $\theta$ indicates that the tail decays quickly (slowly) and, therefore, is thin (fat). Such tail shape estimator and tail index give us a good representation of the curvature of the tails, but since tails may have the same shape while estimating diverse extreme observations, we also employ the semi-parametric extreme quantile estimator from De Haan et al. (1994):

$$
\hat{q}_{p}=x_{k+1}\left(\frac{k}{p n}\right)^{\frac{1}{\hat{\theta}}}
$$

where $n$ the sample size, $p$ is a corresponding exceedance probability, which means the likelihood that a return $x_{j}$ exceeds the tail value $q$, and $x_{k+1}$ is the tail cut-off point. We note that one of the input of $\hat{q_{p}}$ is the tail shape estimator $\varphi$. Similar to value-at-risk (VaR) modeling, the $\hat{q}_{p}^{-}$statistic indicates the level of the worst return occurring with probability $p$, which is small. This is the reason why we call $\hat{q}_{p}$ extreme quantile return (EQR). As we are interested only in the upside returns with a $p$ probability estimated from calls, we only compute $\hat{q}_{p}^{+}$by applying the same methodology to the right side of the RND obtained from the single stock option market $^{14}$.

In addition to the EQR, we also evaluate the density function tails using expected shortfall (ES), which captures the average loss beyond the tail cut-off point. As we are interested in the upside of the distribution, we call such measure expected upside (EU) as the average gain beyond the tail cut-off point. We evaluate the EU following Danielsson et al. (2006) formulae for the ES, which relates the EQR (i.e., the VaR) to the ES (i.e., the CVaR) as described below:

$$
\widehat{E U}_{q(p)}=\frac{\hat{\theta}}{\hat{\theta}-1} \cdot x_{k+1}\left(\frac{k}{p n}\right)^{\frac{1}{\hat{\theta}}}
$$

where $\theta$ is the tail index.

De Haan et al. (1994) show that the tail shape estimator statistic $\sqrt{k}(\hat{\varphi}(k)-\varphi)$ and the tail quantile statistic $\frac{\sqrt{k}}{\ln \left(\frac{k}{p k}\right)}\left[\ln \frac{\hat{q}(p)}{q(p)}\right]$ are asymptotically normally distributed. Hence, according

\footnotetext{
${ }^{14}$ Our EQR measure is closely connected to the risk-neutral tail loss measure of Vilkov and Xiao (2013).
} 
to Hartmann et al. (2004) and Straetmans et al. (2008), the $t$-statistics for such estimators are given by:

$$
T_{\varphi}=\frac{\hat{\varphi}_{1}-\hat{\varphi}_{2}}{\sigma\left[\hat{\varphi}_{1}-\hat{\varphi}_{2}\right]} \sim N(0,1)
$$

and

$$
T_{q}=\frac{\hat{q_{1}}-\hat{q_{2}}}{\sigma\left[\hat{q_{1}}-\hat{q_{2}}\right]} \sim N(0,1)
$$

where the denominators are calculated as the bootstrapped difference between the estimated shape parameters $\varphi$ and the quantile parameters $\hat{q_{p}}$ using 1000 bootstraps. The null hypothesis of this test is that $\hat{\varphi}$ and $\hat{q_{p}}$ parameters do not come from independent samples of normal distributions, therefore, $\hat{\varphi}_{1}=\hat{\varphi}_{2}$ and $\hat{q_{1}}=\hat{q_{2}}$. The alternative hypothesis is that $\hat{\varphi}$ and $\hat{q_{p}}$ have unequal means. Such $t$-test is also applied to our EU analysis, as the distribution of EU follows

the same distribution of the tail quantile statistic $\frac{\sqrt{k}}{\ln \left(\frac{k}{p k}\right)}\left[\ln \frac{\hat{q}(p)}{q(p)}\right]$, given that EU is the extreme quantile estimator multiplied by a constant.

\subsection{Estimating RND and EDF}

For the estimation of the RND, the first step taken is the application of the Black-Scholes model to our IV data to obtain options prices $(C)$ for the S\&P 500 index. Once our data is normalized so strikes are expressed in terms of percentage moneyness, the instantaneous price level of the S\&P 500 index $\left(S_{0}\right)$ equals 100 for every period for which we would like to obtain implied returns. Contemporaneous dividend yields for the S\&P 500 index are used for the calculation of $P$ as well as the risk-free rate from three-, six-, and twelve-month T-bills. Because we have IV data for five levels of moneyness, we implement a modified Figlewski (2010) method for extracting our RND structure, as in Felix et al. (2016). The main advantage of this method over other techniques is that it extracts the body and tails of the distribution separately, thereby allowing for fat tails.

The Figlewski (2010) method is close to the one employed by Bliss and Panigirtzoglou (2004), where body and tails are also extracted separately. Bliss and Panigirtzoglou (2004) use a weighted natural spline algorithm for interpolation, which has the same decreasing-noise effect in RNDs of using splines in the absence of knots, as done in Figlewski (2010). The extrapolation in Bliss and Panigirtzoglou (2004) is done by the introduction of a pseudo-data point, which has the effect of pasting lognormal tails into the RND. One advantage of these two approaches is that the extrapolation does not result in negative probabilities, which is possible when splines is applied in such case. Nevertheless, we favor Figlewski's (2010) approach as the lognormal tails employed by Bliss and Panigirtzoglou (2004) assume that IV is constant beyond the observable strikes, resembling the Black-Scholes model. The modification made to the Figlewski (2010) method by Felix et al. (2016) entailed having flexible inner anchor points (as opposed to having fixed anchor points) for fitting tails to the risk neutral density. The aim of this modification is to prevent the method to estimate distribution density functions with 
implausible shapes.

We estimate the EDF in two different ways. First, using the entire sample of realized returns $(r)$, we estimate long-term EDFs non-parametrically, where $r=\ln \left(S_{T} / S_{t}\right)$ and $S_{t}$ is the realized return index at time $t$ and $S_{T}$ is the forward level of the same index three-, six- or twelvemonths forward, i.e., respectively 21, 63 and 252-days forward. Because of overlapping periods, we initially estimate our empirical distribution from non-overlapping returns for these three maturities by using distinct starting points. This methodology is also applied by Jackwerth (2000) and Ait-Sahalia and Lo (2000). However, because the length of the overlapping periods is relatively large compared to our total sample, especially for the twelve-month forward returns, we average the distribution with distinct starting points to smooth the shape of our multiplehorizon distributions ${ }^{15}$.

In a second step, we estimate time-varying EDFs built from an invariant component, the standardized innovation density, and a time-varying part, the conditional variance $\left(\sigma_{t \mid t-1}^{2}\right)$ produced by an EGARCH model (see Nelson, 1991). We first define the standardized innovation, being the ratio of empirical returns and their conditional standard deviation $\left(\ln \left(S_{t} / S_{t-1}\right) / \sigma_{t \mid t-1}\right)$ produced by the EGARCH model. From the set of standardized innovations produced, we can then estimate a density shape, i.e., the standardized innovation density. The advantage of such a density shape versus a parametric one is that it may include, the typically observed, fat-tails and negative skewness, which are not incorporated in simple parametric models, e.g., the normal. As mentioned, such density shape is invariant and it is turned time-varying by multiplication of each standardized innovation by the EGARCH conditional standard deviation at time $t$, which is specified as follows:

$$
\ln \left(S_{t} / S_{t-1}\right)=\mu+\epsilon_{t}, \epsilon \sim f\left(0, \sigma_{t \mid t-1}^{2}\right)
$$

and

$$
\sigma_{t \mid t-1}^{2}=\omega_{1}+\alpha \epsilon_{t-1}^{2}+\beta \sigma_{t-1 \mid t-2}^{2}+\vartheta \operatorname{Max}\left[0,-\epsilon_{t-1}\right]^{2},
$$

where $\alpha$ captures the sensitivity of conditional variance to lagged squared innovations $\left(\epsilon^{2}{ }_{t-1}\right)$, $\beta$ captures the sensitivity of conditional variance to the conditional variance $\left(\sigma^{2}{ }_{t-1 \mid t-2}\right)$, and $\vartheta$ allows for the asymmetric impact of lagged returns $\left(\vartheta \operatorname{Max}\left[0,-\epsilon_{t-1}\right]^{2}\right)$. The model is estimated using maximum log-likelihood where innovations are assumed to be normally distributed.

Up to this point, we managed to produce a one-day horizon EDF for every day in our sample but we still lack time-varying EDFs for the three-, six-, and twelve-month horizons. Thus, we use bootstrapping to draw 1,000 paths towards these desired horizons by randomly

\footnotetext{
${ }^{15}$ As a robustness check to this approach, we compare our three-, six- and twelve-month empirical distributions with the ones calculated from non-overlapping returns. We use data since 1871 for the US equity price index, made available by Welch and Goyal (2008), who use S\&P 500 data since 1926, and data from Robert Shiller's website for the preceding period. Our empirical distributions are quite similar to the ones estimated from the longer data set, suggesting that they are, indeed suitable as long-term distributions. The use of overlapping returns is less problematic in our calculations than in regression estimation, where statistical inferences on parameter estimates can be strongly affected by overlapping returns' serial correlation.
} 
selecting single innovations $\left(\epsilon_{t+1}\right)$ from the one-day horizon EDFs available for each day in our sample. We note that once the first return is drawn, the conditional variance is updated $\left(\sigma_{t-1 \mid t-2}^{2}\right)$ affecting the subsequent innovation drawings of a path. This sequential exercise continues through time until the desired horizon is reached. In order to account for drift in the simulated paths, we add the daily drift estimated from the long-term EDF plus the riskfree rate to drawn innovations, thus the one-period simulated returns is $\epsilon_{t+1}+\mu+R f$. The density functions produced by the collection of returns implied by the terminal values of every path and their starting points are our three-, six-, and twelve-month EDFs. These simulated paths contain, respectively, 63, 126, and 252 daily returns. We note that by drawing returns from stylized distributions with fat-tails and excess skewness, our EDFs for the three relevant horizons also imbed such features. Finally, once these three time-varying EDFs are estimated for all days in our sample, we estimate $\gamma$ for each of these days using Eq. $(8)^{16}$.

Our approach for estimating both the long-term EDF and the time-varying EDF is closely connected to the method applied by Polkovnichenko and Zhao (2013). The time-varying method used by these authors is based on Rosenberg and Engle (2002). The choice for an EGARCH approach versus the standard GARCH model is due to the asymmetric feature of the former model that imbeds the "leverage effect" 17.

\section{Empirical analysis and results}

In this section, we present our results of the empirical analysis described in section 3 . We note that since we estimate EDF in the two ways described (the long-term and time-varying EDFs), we are able to estimate long-term $\gamma$ 's and time-varying $\gamma$ 's by minimizing Eq. (8). We use our long-term $\gamma$ estimates to compute the ECPT with the aim to compare it to the other subjective density functions using the tests described in Section 3.3. The time-varying estimates of $\gamma$ are analyzed in Sections 4.3 and 4.4 with the use of a regression model. We describe this regression together with its results in Section 4.4. Finally, in Section 5, we perform robustness tests on our results by using an alternative weighting function to the CPT, the one imbedded in the Prelec (1998) model, and we apply Kupiec's test to probability tails, among other checks.

\subsection{Estimated CPT long-term parameters}

We report the estimated CPT parameters $(\lambda$ and $\gamma)$ extracted from long-term density functions in Table 1, Panel A. Our first finding is that $\lambda$, the parameter of loss aversion, which is 2.25 in the CPT, fluctuates around that number for six- and twelve-month options but shows a quite different outcome for three-month options. Our estimation of $\lambda$ from three-month options is

\footnotetext{
${ }^{16}$ Due to drift, the model of time-varying EDF for the twelve-month horizon occasionally does not match the one of the PCPT model. This difference is challenging to estimation of $\gamma($ Eq. (8)), as a large amount of $\gamma$ estimates produce unreasonable PDFs such as non-monotonic CDFs. Therefore, to perform the optimizations given by Eq. (8), we neutralize the impact of the drift by forcing the mode of the simulated EDF to match the one of the PCPT.

${ }^{17}$ The leverage effect is the negative correlation between an asset's returns and changes in its volatility. For a comparison between alternative GARCH approaches, see Bollerslev et al. (2009).
} 
1.02, which indicates no loss aversion. For the six- and twelve-month options $\lambda$ is 2.66 and 3.00, respectively. This finding suggests that loss aversion is more pronounced at longer maturities than suggested by the CPT. Apart from that, twelve-month $\lambda$ estimates are highly variant across the different optimization upper bounds used (i.e. 3, 5 and 10), always matching the bound value, whereas estimates from three- and six option maturities are very stable across upper bounds.

[Please insert Table 1 about here]

The estimated probability weighting function parameter $\gamma$ is slightly higher than the one suggested by the CPT (i.e., 0.61) at the three- and six-month horizons, respectively, at 0.75 and 0.81. For twelve-month options, $\gamma$ is around 1.09. These results suggest that overweighting of small probabilities occurs in short-term options (up to six-months), while twelve-month options seem to behave more rationally. These findings support our hypothesis that individual investors are, on average, biased when purchasing single stock call options, as suggested by Barberis and Huang (2008).

\subsection{Density functions tails' consistency test results}

As specified in section 3.3, we test the empirical consistency of density function tails among a set of five subjective distributions (CPT, PCPT, ECPT, power, exponential), the RND, and the EDF. We perform these tests by employing EVT through the application of Eqs. (9) to (12b). For such purpose, we require return streams $\left(x_{j}\right)$, which are only available for the longterm EDF. Thus, we apply an inversion transform sampling technique to our other PDFs to obtain sampled returns for them. Such method, also known as the Smirnov method, entails drawing $n$ random numbers from a uniformly distributed variable $U=\left(u_{1}, u_{2}, \ldots, u_{n}\right)$ bounded at interval $[0,1]$ and, subsequently, computing $x_{j} \leftarrow F^{-1}\left(u_{j}\right)$, where $F$ are the CDFs of interest (see Devroye, 1986, p.28). Hence, the Smirnov method simulates returns that resemble the ones of the inverse CDF by randomly drawing probabilities along such function.

Once we obtain returns for all five PDFs, the next step is to set $k$ as the optimal number of observations used for estimation of $\varphi$ by Eq. (9), the Hill-estimator. For this purpose, we produce Hill-plots for the right tail of our distributions, which depict the relationship between $k$ and $\varphi$ as a curve (see Straetmans et al., 2008). Picking the optimal $k$ is done by observing the interval in this curve where the value of $\varphi$ stabilizes while $k$ changes. This area suggests a stable trade-off between a good approximation of the tail shape by the Pareto distribution and the uncertainty of such approximation (by the use of fewer observations). The interval that corresponds to roughly four to seven percent of observations seems to be a stable region across the Hill-plots of the tails of the EDF and the CPT. As an increase in $k$ increases the statistical power of the estimator but may distort the shape of the tail, we decide to set $k$ as chosen from the Hill-plots for EDF and CPT tails equal to four percent.

We examine whether the tail shape parameter $(\varphi)$, computed via the Hill (1975) estimator, for the RND and for our subjective density functions (i.e., power, exponential, PCPT, CPT and ECPT) matches the one for the EDF. The outcomes from the statistical tests performed 
to compare tail shape parameters (Eq. (12a)) are provided in Table 1, Panel B. Results suggest that for the three-month maturity options, $\varphi$ for the RND, CPT and ECPT (at 0.20) are the closest to the EDF parameter (at 0.29 ) but they are not statistically equal. The $\varphi$ estimate for the power, exponential, and PCPT density functions do not match the one for the EDF, as they are all around 0.17 and, thus, exhibit fatter tails than the EDF.

We observe that the results for the six- and twelve-month options are very similar to the ones obtained for the three-month expiry. The parameter estimate $\varphi$ of the EDF is statistically equal to the RND and CPT. Parameter $\varphi$ ranges from 0.18 to 0.19 for the CPT, ECPT, and RND for the six- and twelve-month maturities, whereas it is 0.23 for the EDF. The estimate of $\varphi$ for the RND (0.19 and 0.22 for the six- and twelve-month maturities, respectively) somewhat matches the one for the EDF at the six-month maturity but it is off at the twelve-month maturity. The parameter estimates $\varphi$ for the power, exponential, and PCPT density functions match the EDF's $\varphi$ at the twelve-month maturity only. Generally, the parameter estimates $\varphi$ for these subjective density functions are too small in comparison to the one of the EDF. This means that these six- and twelve-month maturity subjective density functions have fatter tails than the EDF, the other subjective densities (CPT and ECPT), and the RND. These results suggest that the shape of the CPT density function is a good match to the shape of realized tails.

After $k$ is chosen and the shape estimator $\varphi$ for the EDF, RND, power, exponential, PCPT, CPT, and ECPT is computed, extreme quantile returns (EQR) can also be estimated via Eq. (10). Subsequently, the $t$-test in Eq. (12b) is applied using the one, five and ten percent statistical significance levels. This test evaluates whether the EQRs estimated from a set of two distributions (RND, power, exponential, PCPT, and CPT versus EDF) have equal means (the null hypothesis). The results of this test are shown in Table 2, Panel A.

[Please insert Table 2 about here]

Analyzing the density functions derived from the three-month option maturity, we find that the EQR implied by the CPT is the only one that matches the realized EQR and at the first quantile solely at 21 percent. The EQR implied by the ECPT is almost the same as implied by the CPT, thus, it also statistically matches the EDF. Per contrast, the EQRs for the RND, power, exponential, and PCPT densities always overshoot the one for the EDF. All comparisons between these distributions' EQR at the three-month maturity reject the null hypothesis that returns at the same quantile are equal. This pattern is observed across all quantiles analyzed, i.e., at the tenth, fifth, and first quantiles. This empirical finding indicates that the equity market upside implied in option markets (i.e., the RND) and the power, exponential and PCPT densities are always higher than the ones realized by the equity market. The results for the PCPT resemble the ones for the RND. The EQRs from the CPT and the ECPT are clearly the best matches for the EDF.

For the six-month maturity, upside returns priced by the RND and ECPT best match the EQR. The EQRs for the EDF are roughly 18, 22, and 32 percent for the tenth, fifth, and first quantile of returns, respectively, whereas the EQRs for the ECPT are 19, 21, and 28 percent. 
For the RND, such extreme upside return estimates are 19, 22, and 30 percent. Thus, the ECPT statistically matches the realized EQR best at the tenth and fifth quantile, whereas the RND is the best match for the third quantile. No rational subjective density function consistently matches the EQR of the EDF. The power, exponential, and PCPT densities almost always overshoot the EQR of realized returns. Per contrast, the CPT density always undershoots the EDF's extreme returns. Despite always overshooting the EQR of the EDF, the PCPT is the only other subjective density (apart from the ECPT) that has EQR statistically equal to the EDF, which happens only at the first quantile EQR.

In contrast to the three- and six-month maturities, the EQRs from the RND for the twelvemonth maturity all underestimate the EQRs from realized returns. The EQRs of realized returns are 32, 35, and 44 percent for the tenth, fifth and first quantiles, respectively, whereas for the RND these are 22, 26, and 37 percent, respectively. The same underestimation is documented for the densities linked to the CPT (i.e., PCPT, CPT and ECPT) as tail returns are largely out of sync with realized ones, especially for the CPT in which overweight of tails will force EQRs further away from EDF ones (vis-à-vis the PCPT EQRs). The EQRs of the exponential densities continue to largely overshoot the ones for the EDF. However, the power utility function density successfully matches the EQR returns across all EQR values and with strong statistical significance.

In line with these results for the EQR, Table 2, Panel B, shows that the expected upside (EU) for the EDF is more closely matched within the three-month horizon by the CPT and ECPT density functions for the tenth, fifth and first quantiles. The three-month horizon EUs estimated from the realized returns are 15, 19, and 30 percent for the mentioned quantiles. The ECPT EUs for the same horizon are 18, 21, and 28 percent, respectively. For the CPT, EUs are 16, 19 and 26 percent. Thus, estimates from these two density functions are mostly statistically equal to the realized returns. Similarly to our analysis on the EQR, for the other subjective densities, the EUs for all quantiles are also much larger than the EDF expected upside. The exponential density has the highest expected upside across the different quantiles, being the furthest away from the realized returns. The RND-implied expected upside is somewhat conservative and relatively closer to the realized ones but only statistically significant at the one percent quantile.

For the six-months maturity, the expected upsides for the CPT and ECPT density functions are no longer that close to each other nor to the realized ones. The EDF expected upside always exceeds the ones for the CPT and ECPT. Only at the tenth quantile, the expected upside of the ECPT density function equals the realized one. The densities which better match the expected upside of the EDFs are the PCPT and the RND.

For the twelve-month horizon, the expected upside for the realized returns is 37,40 , and 51 percent for the tenth, fifth and first quantiles. In line with the results from our EQR analysis, the power density again best matches realized EUs, as estimates are statistically equal across all maturities. Second best performers are the PCPT and ECPT densities, which match the realized EU at the one percent quantile level.

In summary, across the three EVT tests performed (i.e., on tail shape, EQR and EU), the 
three option maturities and the three quantiles evaluated, we observe that the success rate of the CPT subjective density functions on matching the EDF tails is 57 percent. In contrast, this success rate is 38 percent for the power utility, 33 percent for the RND and only 10 percent for the exponential utility density function. These results suggest that CPT-related distributions, although not always matching the EQRs and ES of the EDF, seem to best match the EQR of the EDF, especially at the short maturities. More specifically, the ECPT seems to have some advantage over the other methods for the three- and six-month maturities. This results is consistent with the findings of Kang and Kim (2006), who find that more flexible utility functions generally increases the forecasting ability of subjective PDFs. In the case of the CPT, the fact that its weighting function can assume different shapes does entails extra flexibility to match the data relative to traditional utility functions. Thus, if our findings suggest that the CPT does not fully explain single stock options pricing, its overweighting of small probabilities feature goes very far in explaining such market data, with the exception of twelve-month options.

These findings reiterate our takeaway from Section 4.1, in which a positive term structure of overweight of tails appears to play a substantial role: twelve-month options are priced more rationally than shorter term ones, which seem to be priced as a result of lottery buying by individual investors. Figure 2 compares the CDFs from six of our equity return densities: the EDF, the RND, the CPT, the PCPT, the exponential- and the power-utility density ${ }^{18}$. We focus on the right tails of these distributions as we are interested in how closely the RND from call options and derived subjective density functions match the tails of the EDF. The plots display the cumulative probabilities on the $\mathrm{y}$-axis and the terminal price levels on the $\mathrm{x}$-axis, given an initial price level of 100 .

[Please insert Figure 2 about here]

In Figure Figure 2, we see that the tails implied by option prices (RND, in red) seem fatter than the tails from the CPT (in dark blue) and EDF (in green) density functions over the three-month horizon. The tails for the CPT and the EDF are almost identical above the 120 terminal level, i.e., at the 20 percent return. The right tail of the RND distribution is clearly much fatter than the ones of the CPT and EDF, but it is still thinner than the ones of the PCPT, the exponential- and the power-utility densities. Thus, the upside risk implied from options is much higher than the one realized by the EDF, a sign of a potentially biased behavior by investors in such options. This observation is confirmed by the tail shape parameter $(\varphi)$, the EQRs and the EU estimated across the different quantiles, which in all cases report higher upside in the RND than in the EDF and the CPT. Figure 2 also suggests that the upside risk of the RND is more consistent with the PCPT density, whereas the CPT tails seem very distinct from the PCPT, which is in line with our earlier findings.

The plot in column B, which depicts the CDF for our studied densities at the six-month horizon, suggests that the RND and the EDF are closer than at the three-month horizon.

\footnotetext{
${ }^{18}$ We omit the ECPT for better visualization as its CDFs are very similar to the CPT ones. The similarity is caused by the ECPT left tail weighting function parameter $(\delta)$ being the same for the CPT and because the estimated long-term $\gamma$ for the three maturities are close to the Tversky and Kahneman (1992) one.
} 
At the same time, the CPT density seems more disconnected from the EDF. This finding matches our results from the EQR and the expected upside comparisons. The PCPT tail is, at this horizon, higher than the EDF, CPT, and RND ones and closer to the EDF one than to the CPT one, especially at its very extreme. This finding is also confirmed by our EQR and expected upside tests, as the PCPT is statistically equal to the EDF at the one percent quantile. The exponential and power utility densities have right tails that are much fatter than the other densities, including the EDF.

Figure 2 shows that at the twelve-months horizon the CPT's CDF tails seem completely disconnected from the EDF. The EDF tails are much fatter than the CPT ones and slightly fatter than the RND ones. In fact, the RND seems to match the EDF for terminal levels above 120. This finding suggests that long-term options trade in a much less CPT-biased manner than short-term options.

Overall, the visual inspection of our density function CDFs confirms our hypothesis that end-users of OTM single stock calls are likely biased and behave as buying lottery tickets when trading short-term options. These results strengthen the evidence provided by Ilmanen (2012), Barberis (2013), Conrad et al. (2013), Boyer and Vorkink (2014) and Choy (2015) that investors push single stock options prices to extreme valuation levels. Investors seem to overweight small probabilities especially at short-term horizons. Next, we analyze the timevariation in overweight of small probabilities to better understand the underlying reasons for our findings.

\subsection{Estimated CPT time-varying parameters}

To investigate time-variation in the CPT's overweighting of small probabilities in single stock options, we apply Eq.(8) to each day in the sample to estimate the empirical $\gamma$ (weighting function) parameter. Lower and upper bounds of -0.25 and 1.75 were used in this optimization as they produced the lowest RSS across permutation of all bounds when $\gamma$ was optimized using the CPT parameterization. We estimate $\gamma$ under four different assumptions about $\lambda$, the loss aversion parameter: 1) $\lambda$ equals 2.25 , the CPT parameterization; 2) no loss aversion, $\lambda$ equals 1; 3) augmented loss aversion, $\lambda$ equals 3 ; and 4) optimal $\lambda$, as estimated by Eq.(7).

Table 3, Panel A reports the statistics when $\lambda$ equals 2.25. We find that the median and the mean time-varying values of $\gamma$, estimated from the three-month options are above its CPT value of 0.61 but still reflect overweight of small probabilities. This suggests that overweighting of small probabilities is present within the pricing of three-month call options as suggested by the theory. The distribution of $\gamma$ is skewed to the right and overweight of small probabilities is present 64 percent of times within three-month maturity. The $25^{\text {th }}$ percentile of $\gamma$ is 0.74 , clearly suggesting a less pronounced overweight of small probabilities than suggested by the CPT. The estimates of $\gamma$ range from 0 to 1.75 (i.e., an underweighting of small probabilities) and are volatile, with a standard deviation of 0.23 . Interestingly, when we split the sample in three parts (as shown in Table 3), we observe that overweight of small probabilities is very present at the beginning of our sample, in 97 percent of the days from 1998-01-05 to 2003-01-30, 
but that has faded since 2003. During the period from 2003-01-31 to 2008-02-21, underweight of small probabilities is present in 65 percent of the days, whereas such condition is less pervasive from 2008-02-22 onwards, i.e., until 2013-03-19. This finding suggests that overpricing of single stock options is sample specific and not structural. Even if sample specific, overweight of small probabilities seems, in general, much less pronounced than the 0.61 parameter offered by the CPT. These results seem to only partially confirm our hypothesis that the CPT can empirically explain the overpricing of OTM single stock call options.

[Please insert Table 3 about here]

At the six-month maturity, overweighting of small probabilities is less frequent than in threemonth tenor. The median $\gamma$ for such maturity is 0.99 , implying roughly neutral probability weighting. The long-term $\gamma$ equals 0.81 and is somewhat out-of-sync with the time-varying estimates. Similarly to the three-month maturity, the distribution of $\gamma$ is also slightly skewed to the right. The $75^{\text {th }}$ quantile of $\gamma$ equals 1.14 and suggests an underweighting of tail probabilities. However, probability weighting is largely sample dependent as within the overall sample, 52 percent of all observations reflect overweight of small probabilities but, between 1998 and 2003, its occurrence is 92 percent.

Differently from the other maturities, $\gamma$ estimates for the twelve-month maturity tend towards underweight of tail probabilities. The median $\gamma$ is 1.03, whereas the mean $\gamma$ is 1.01. Time variation and sample dependence are present as for the other maturities but, at the twelve-month maturity, the percentage of days with overweight of tails is smaller, 41 percent in the full sample but still 83 percent for the 1998-2003 sample.

In summary, the statistics in Table 3 , Panel A, indicate that the weighting function parameters $\gamma$ for the three maturities evaluated are time-varying and sample specific. Overweight of small probabilities holds for the three-month maturity, less convincingly so for the six-month maturity, and not at all for the twelve-month maturity, in which neutral probabilities and underweight of tails respectively prevails.

Because the loss-aversion parameter $\lambda$ is of high importance in the CPT model, we estimate $\gamma$ under different $\lambda$ parameterizations, more specifically, for 1) $\lambda$ equals 1,2$) \lambda$ equals 3 and optimal $\lambda$, as estimated from the long-term empirical distribution (see Table 1).

We report the summary statistics of the new $\gamma$ estimates in Panel B of Table 3, when we assume $\lambda$ equals 1 . The new median and mean estimates for $\gamma$ are 0.66 and 0.67 for the threemonth maturity, respectively, and, thus, lower than when $\gamma$ was estimated under the CPT loss aversion calibration $(\lambda=2.25)$. The $75^{\text {th }}$ percentile of $\gamma$ also decreases, from 1.14 to 0.80 . At the six-month horizon, the difference between $\gamma$ with $\lambda$ equals 2.25 and with $\lambda$ equals 1 is also large. The median $\gamma$ for the CPT $\lambda$ is 0.99 , whereas for when $\lambda$ equals 1 it is 0.71 . The means are 0.96 and 0.72 , respectively. At the $75^{\text {th }}$ percentile using $\lambda$ equals $1, \gamma$ becomes 0.87 . For the twelvemonth maturity, we observe a similar effect. The median $\gamma$ for when $\lambda$ equals 1 is 0.83 , whereas for when $\lambda$ equals 2.25 it is 1.03. In brief, a lower loss aversion parameter consistently gives rise to higher $\gamma$ estimates, across the different options' maturities and quantiles. The opposite effect is observed when the $\lambda$ is increased from 2.25 to 3 , as shown by Table 3, Panel C. The 
median and mean $\gamma$ when $\lambda$ equals 3 becomes 0.96 and 0.98 for the three-month maturity, in comparison to 0.91 and 0.89 when $\lambda$ equals 2.25. Such rise in central tendency of $\gamma$ estimates is also observed within the six- and twelve-month maturities and across the 25 and 75 percent quantiles. Table 3, Panel D, which reports $\gamma$ estimates when optimized $\lambda$ parameters are used, shows distinct results for the three-month maturity versus the six- and twelve-month maturity. For the three-month maturity, we observe a downward shift to $\gamma$ estimates, whereas for sixand twelve-month maturities, an upward movement in estimates occurs. However, this initially opposite effect in estimates is, in fact, qualitatively equal to the result just described when we use $\lambda$ as 1 or 3 , as the optimal $\lambda$ parameters estimated for the three-, six and twelve-month maturities are, respectively, 1.02, 2.66 and 3.00 (i.e., it decreases for the three-month maturity and increases for the six- and twelve-month maturity vis-à-vis the CPT parameterization).

The reason why a lower (higher) loss aversion gives rise to a decreased (increased) $\gamma$ is that it increases (decreases) the probability on the left side of distribution, influencing the probabilities and the shape of the right side of the CPT distribution. High values of $\lambda$ push the CPT density to have more probability on the right side of the distribution, which is spread proportionally to the probabilities originally observed in the right-side bins (i.e., creating a bump into the centerright side of the distribution), all else equal. Thus, the impact of such probability shift fades as the tail approaches. Nevertheless, the right tail of the CPT density does turn fatter (and the $\gamma$ parameter higher) as $\lambda$ is made higher. The opposite occurs if low values of $\lambda$ are assumed: the right tail of the CPT density becomes thinner, causing $\gamma$ estimates to be low (which more forcefully can turn the RND right tail into such thin CPT tail). One important finding from our experimentation with different $\lambda$ parameters is that the time variation observed when $\lambda$ equals 2.25 is unchanged. The standard deviation and range of $\gamma$ estimates across the use of the different $\lambda$ values are somewhat the same. Though, the percentage of days that overweight of tails is observed in the different samples studied dramatically changes towards a more frequent presence of overweight of small probabilities, as low levels of $\lambda$ are used (and vice-versa). The large difference in the presence of overweight of small probabilities across samples remains.

We interpret our finding that $\gamma$ is strongly time-varying and sample dependent across all maturities and under different $\lambda$ assumptions as a strong evidence that single stock options are not overvalued due to a structural skewness preference, as Barberis (2013) may suggest. We reckon that, if static skewness preferences would drive overweight of small probabilities, parameter $\gamma$ would be relatively stable throughout our sample. Given that the $\gamma$ is largely volatile, we support the view that investors experience (time-varying) "bias in beliefs" or, alternatively, time-varying preferences (see Barberis, 2013) ${ }^{19}$. Our results are in line with Green and Hwang (2011), Chen et al. (2015) and Jiao (2016), who report similar time-varying effects in the over-

\footnotetext{
${ }^{19}$ Barberis (2013) distinguishes investors' time-varying beliefs from skewness preferences as he argues that investors with biased beliefs mistakenly overestimate tail events, whereas preference for skewness leads to overweight of tails, which is less likely to be a mistake. As an example, the author suggests that investors that overweight small probabilities events correctly anticipate the distribution of a stock's future returns but overweight the state of the world in which a stock turns out to be "the next Google". In the example, overestimation of tail events would occur when the investor attributes a higher chance to the stock being the next Google. As we do not attempt to distinguish between biased believes and time-varying preferences, we use the term overweight of small probabilities throughout our paper.
} 
pricing, skewness effects and returns for IPOs and lottery-like stocks. These papers also report that, beyond time-varying effects, stronger skewness preferences are associated with higher participation of individual investor (trading in IPOs, trading around earnings announcements and owning stocks) in detriment of institutional investors.

\subsection{Time variation in probability weighting parameter and investors' sentiment}

As observed in section 4.3, the probability weighting parameter $\gamma$ is clearly time-varying. In the following, we investigate which factors may explain this time-variation of $\gamma$. Our main hypothesis is that it is linked to investor sentiment. The link between sentiment and overweighting of small probabilities or lottery buying in OTM single stock calls originates from the fact that individual investors are highly influenced by market sentiment and attention-grabbing stocks (Barberis et al., 1998; Barber and Odean, 2008; Berger and Turtle, 2015), and that OTM single stock calls trading is speculative in nature and mostly done by individual investors (Lakonishok et al., 2007). For instance, Lakonishok et al. (2007) argue that the IT bubble of 2000, a period of high variation of $\gamma$, is linked to elevated investor sentiment, when the least sophisticated investors were the ones most inclined to purchase calls on growth and IT stocks. Figure 3 depicts time-varying $\gamma$ 's and the Baker and Wurgler (2007) sentiment factor. It provides evidence that these measures move in tandem at times. For example, during the IT bubble, the level of $\gamma$ seems quite connected with the level of sentiment, especially for the three- and six-month options.

\section{[Please insert Figure 3 about here]}

To formally test our hypothesis that time variation of $\gamma$ is linked to investor sentiment, we design a regression model. In Eq. (14) the explained variables are $\gamma$ for the three-, six-, and twelve-month horizons and the explanatory variables are the Baker and Wurgler (2007) sentiment measure ${ }^{20}$; the percentage of bullish investors minus the percentage of bearish investors given by the survey of the American Association of Individual Investors (AAII), used as a proxy for individual investor sentiment by Han (2008); and a set of control variables among the ones tested by Welch and Goyal $(2008)^{21}$ as potential forecasters of the equity market. The data frequency used in the regression is monthly as this is the highest frequency available from the sentiment data and from the Welch and Goyal (2008) data set ${ }^{22}$. Our regression sample starts in January 1998 and ends in December 2010²3. Our OLS regression model is specified as

\footnotetext{
${ }^{20}$ Available at http://people.stern.nyu.edu/jwurgler/.

${ }^{21}$ The complete set and description of variables suggested by Welch and Goyal (2008) is provided in Appendix B. From the complete set of variables used by Welch and Goyal (2008), we select a smaller set using the crosscorrelation between them to avoid multicollinearity in our regression analysis. Because we run a multivariate model, using the full set of variables is undesirable as some of them correlate 80 percent with each other. We exclude variables that correlate more than 40 percent with each other.

${ }^{22}$ Given the fact that $\gamma$ is estimated on a daily basis, we average $\gamma$ throughout each month.

${ }^{23}$ This regression sample is only possible because Welch and Goyal (2008) updated their dataset after the paper publication. The regression sample however, could not be extended further than December 2010 because the sentiment measure of Baker and Wurgler (2007) is not available after that date.
} 
follows:

$$
\begin{array}{r}
\gamma_{t}=c+\psi_{1} \cdot \text { Sent }_{t}+\psi_{2} \cdot \text { IISent }_{t}+\psi_{3} \cdot E 12_{t}+\psi_{4} \cdot B / m_{t}+\psi_{5} \cdot N \text { tis }_{t}+ \\
\psi_{6} \cdot \text { Rfree }_{t}+\psi_{7} \cdot \text { Infl }_{t}+\psi_{8} \cdot \text { Corpr }_{t}+\psi_{9} \cdot \text { Svar }_{t}+\psi_{10} \cdot C S P_{t}+\epsilon_{t}
\end{array}
$$

where Sent is the Baker and Wurgler (2007) sentiment measure, IISent is the AAII individual investor sentiment measure, E12 is the twelve-month moving sum of earnings on the S\&P5000 index, $B / m$ is the book-to-market ratio, Ntis is the net equity expansion, $R$ free is the risk-free rate, Infl is the annual inflation rate, Corpr is the corporate spread, Svar is the stock market variance and, $C S P$ is the cross-sectional premium.

Additionally, we run univariate models for each explanatory factor to understand the individual relation between $\gamma$ and the control variables:

$$
\gamma_{t}=c_{i}+\psi_{i} \cdot x_{i, t}+\epsilon_{t}
$$

where $x$ replaces the $n$ explanatory variable earlier specified, given $i=1 \ldots n$.

[Please insert Table 4 about here]

Table 4, Panel A presents the estimates of Eq. (14). We note the high explanatory power of the multivariate regression, ranging from 68 to 71 percent. As expected, we observe that Sent is consistently negative and statistically significant across the three different horizons studied. On average, each one-unit difference in Sent is linked to roughly -0.1 difference in $\gamma$, all else being equal. The univariate regressions of Sent confirm the negative link between sentiment and $\gamma$. For all option maturities, a negative relation between the Baker and Wurgler (2007) sentiment measure and $\gamma$ is clearly found. The explanatory power of the variable Sent in the univariate setting is also high, between 22 and 29 percent. These findings altogether support our hypothesis that overweighting of small probabilities increases at higher levels of sentiment and that sentiment strongly impacts the probability weighting bias of call option investors.

In contrast with the variable Sent, the coefficients for the individual investor sentiment (IISent) are positive but not statistically significant either on the multivariate setting or on the univariate one (see Table 4). The univariate regressions run on $\gamma$ have rather low explanatory power. The positive relationship between IISent and $\gamma$ at the three-month maturity may be attributed to potential capitulations in individual investor sentiment, as such indicator is strongly mean-reverting.

The nine Welch and Goyal (2008) control variables used in our multivariate regression are linked to $\gamma$ in very distinct manners. First, it is fair to say that they add substantial explanatory power to our multivariate regressions. The three-, six-, and twelve-month multivariate models explain, respectively, 71, 68, and 67 percent of the level of $\gamma$. Most of these relations are stable, because the coefficient signs change only rarely. The control variables that are statistically significant in our multivariate setting are E12, B/m, Rfree, Infl, Svar, and CSP (Table 4). We observe that $\gamma$ is positively linked to $E 12$, the twelve-month moving sum of earnings on the 
S\&P 500 index, as well as to $B / m$, the book to market ratio, in both multivariate and univariate regressions. The positive relation between $E 12, B / m$ and $\gamma$ could be explained by meanreversion of earnings and valuation being linked to a greater overweighting of small probabilities, which could be justified by the higher investor sentiment outweighing earning downgrades and rising valuations in a rallying market. These two variables have high explanatory power of $\gamma$, respectively, 37 and 31 percent for the three-month horizon. The significance of $R f r e e$ is, however, somewhat unstable. At the three- and six-month maturity at the multivariate regression $R$ free is significant but not at the univariate regression. Further, the stock market variance, Svar, is negatively linked to $\gamma$. Apparently, the higher the risk environment, the higher the overweighting of small probabilities is. In a univariate setting (at the three-month horizon), the explanatory power of such univariate regression is 18 percent, thus relatively high. Table 4, Panel B indicates that the cross-sectional premium CSP is positive and statistically significant in the univariate setting for the three-month horizon, despite being negative and not significant in the multivariate regressions.

To reiterate our results, we also apply the Least Absolute Shrinkage and Selection Operator (Lasso) methodology to our main multivariate regressions (see Tibshirani, 1996, and Appendix A.3). We apply Lasso to select the regressors that are most relevant for the overall fit of the $\gamma$ by our sentiment and control variables. The coefficients that shrink to zero via the Lasso are identified in Table 4 (Panel A) with a dagger $(\dagger)$. Model selection via the Lasso confirms that Sent and IISent are more relevant for the overall fit of $\gamma$ than some of the fundamental factors used, namely, Ntis, Infl, Corpr and CSP.

The results provided by our OLS regression and by the Lasso indicate that supportive fundamental data for equity markets do not necessarily intensify biased behavior of single stock call option investors. This is an interesting takeaway, especially considering the notion that sentiment does appear to affect such behavior: single stock option investors seem to overweight small probabilities when sentiment is exuberant, not necessarily when stock fundamentals are exuberant.

More importantly, these results support our earlier findings that overweight of small probabilities is strongly time-varying and linked to sentiment. Therefore, overweight of small probabilities is unlikely to result from (static) investor preferences but from investors' bias-in-beliefs or time-varying preferences, which seem conditional on sentiment levels. Furthermore, we also run our regression models (Eqs. (14) and (15)) using different assumptions about the value of $\lambda$, the loss aversion parameter. In this exercise we set $\lambda$ to imply 1$)$ no loss aversion $(\lambda=1)$, 2) augmented loss aversion $(\lambda=3)$ and 3) optimal loss aversion, where $\lambda$ assumes the estimated value by Eq. (7) and reported in Table 1, Panel A.

Table 5 indicates that the results for Sent are similar to the ones obtained in our main regressions: Sent is negatively linked to $\gamma$ and statistically significant at all horizons but with less statistical significance, explanatory power and magnitude at the twelve-month horizon. This result applies to the multivariate regression model only. Across all options maturities, the Sent coefficients become larger when $\lambda$ equals 3 and they shrink when $\lambda$ equals 1 . The 
relation between changes in $\lambda$ and Sent observed is intuitive. We argue that as $\lambda$ increases, the probabilities on the left side of the CPT distribution increase, favoring a thinner tail on the right side of the PCPT distribution, which, then, requires less overweight of tail adjustment (through a higher $\gamma$ ) for the PCPT to match the EDF. As a higher $\gamma$ is obtained by such increase in $\lambda$, the coefficient of $\gamma$ with the given sentiment factor also increases in magnitude. The explanatory power of these regressions are, once again, high, as $R^{2}$ ranges from 62 to 73 percent in the multivariate models. The explanatory power of Sent ranges from 16 to 24 percent in the univariate setting. Table 5 reiterates the relation between IISent, the AAII individual investor sentiment measure, the Welch and Goyal (2008) control variables and $\gamma$ in our main regressions. IISent is rarely significantly linked to $\gamma$. The control variables that are robustly linked to $\gamma$ in our main regression $(E 12, B / m$ and Svar) remain strongly connected to it within these auxiliary regressions. Applying the Lasso model selection technique to these regressions gives results that are analogous to these ones. Sent, IISent, E12, B/m, Svar and $R$ free always survive the Lasso variable selection procedure, whereas Ntis, Infl, Corp and $C S P$ coefficient often shrink to zero (as in our main regression, these coefficients are identified with a dagger $(\dagger)$ in Table 5 , Panel A).

[Please insert Table 5 about here]

The robustness of the relation between $\gamma$ and Sent suggests that changes in the overweighting of tails are not conditional on the level of the loss aversion parameter. In other words, levels of loss aversion do not drive investors to overweight upside tail events, as one could hypothesize when associating upside speculation with a state of low loss aversion. Thus, our results suggest that overweighting of small probabilities is a phenomenon stably linked to sentiment, rather than positive fundamentals or loss aversion levels. Our results tie closely with the findings of Green and Hwang (2011), who investigate the relation between IPOs expected skewness and returns. They find that the skewness effect is stronger during period of high investor sentiment. In the same line, Chen et al. (2015) conclude that when gambling sentiment is high, stocks with lottery-like characteristics earn positive abnormal returns in the short-run followed by underperformance in the long run.

\section{Robustness tests}

\subsection{Kupiec's test for tail comparison}

We employ Kupiec's (1995) test to compare the tails of the EDF with the ones of the subjective density functions and of the RND as a robustness test to the EVT methods applied. Kupiec's test was originally designed to evaluate the accuracy of Value-at-risk (VaR) models, where the estimated VaR were compared with realized ones. Because the VaR is no different from the EQR on the downside, i.e., the $\hat{q}_{p}^{-}$statistic, we can also make use of Kupiec's method to test the accuracy of the $\hat{q}_{p}^{+}$statistic for subjective densities and the RND on matching realized EQRs. Kupiec's method computes a proportion of failure (POF) statistic that evaluates how often a 
VaR level is violated over a specified time span. Thus, if the number of realized violations is significantly higher than the number of violations implied by the level of confidence of the VaR, then such a risk model or consistency of tails is challenged. Kupiec's POF test, which is designed as a log-likelihood ratio test, is defined as:

$$
L R_{P O F}=-2 \log \left[\left(1-p^{*}\right)^{(n-v)}\left(p^{*}\right)^{v}\right]+2 \log \left[\left(1-\left[\frac{v}{n}\right]\right)^{(n-v)}\left(\frac{v}{n}\right)^{v}\right] \sim \chi^{2}(1),
$$

where $p^{*}$ is the POF under the null hypothesis, $n$ is the sample size, and $v$ is the number of violations in the sample. The null hypothesis of such test is $\frac{v}{n}=p^{*}$, i.e., the realized probability of failure matches the predicted one. Thus if the $L R$ exceeds the critical value, $\chi^{2}(1)=3.841$, the hypothesis is rejected at the five percent level. In our empirical problem, $p^{*}$ equals the assumed probability that the EQR of the subjective and risk-neutral densities will violate the EQR of the realized returns, whereas $\frac{v}{n}$ is the realized number of violations. Because we apply Kupiec's test to upside returns, violations mean that returns are higher than a positive threshold.

The first step in applying Kupiec's test to our data set is outlining the expected percentage of failure $\left(p^{*}\right)$ between the EQR from the EDF and from the subjective and risk-neutral densities. We pick $p^{*}$ as being five and ten percent. The percentages can be seen as the expected frequency that the tails of the subjective and of the RND distributions overstate the tails of the distribution of the realized returns. As a fatter tail is a symptom of an overweighting of small probabilities, we expect that densities that do not adjust for the CPT weighting function will deliver a higher frequency of failures than the CPT density function. The Kupiec's test results are reported in Table 6.

[Please insert Table 6 about here]

Panel A in Table 6 suggests that the probability of failure for the RND, power, exponential, and PCPT densities is particularly high at the three-month horizon, with more than 99 percent for the EQR at 90 and 95 percent and for $p^{*}$ equal to five and ten percent. These densities often contain fatter tails than the EDF. For the CPT density, the POF is much lower across the two values of $p^{*}$ used and the 90 and 95 percent EQR. The POF for the 90 percent EQR is roughly 58 percent for the $\mathrm{CPT}$, irrespective of $p^{*}$. At the 95 percent EQR, the POF is 46 percent for the CPT. These findings suggest that at the 90 and 95 percent EQR, the CPT densities overstate less frequently the EDF tails than other densities. The violations of the EDF tails are, however, still significant as they occur between 41 and 52 percent of times. Nevertheless, when we analyze the 99 percent EQR, we find that the POF for all densities decreases considerably and, for the CPT, it becomes 16 percent.

Panel B of Table 6 depicts a very similar pattern of the POF for the probability densities derived from the six-month options as we find for the three-month options. The POF is very close to 100 percent for all densities apart from the CPT at the 90 and 95 percent EQR, while at the 99 percent EQR violations fall substantially, even more than what we observed for the three-month options. Nevertheless, the CPT remains the best approximation for the EDF, as its POF is the lowest. The Kupiec's test result suggests that the CPT density is statistically equal to the EDF, whereas the RND also equals the empirical returns at the ten percent level. 
The results for $p^{*}$ at the five or ten percent are very similar. Panel C presents the POF for the twelve-month maturity. We find once again that the CPT tails are the ones that violate the EDF tails the least. The POF for these densities are about 29 percent for the 90 percent EQR, seven percent for the 95 percent EQR, and four percent for the 99 percent EQR. These findings suggest that the tails of the CPT closely match the EDF ones, especially far out in the tail, i.e., at the 95 and 99 percent EQR. The RND, power, exponential, and PCPT densities record POFs that are much smaller than for the three- and six-month maturities but that are still high in comparison to the CPT.

We note that results for the PCPT and the CPT are quite distinct, whereas results for the PCPT are somewhat closer to the ones of the RND. This suggests that the weighting function is the component within the CPT density function that more forcefully causes the RND to approximate the EDF, so not the value function. Overall, our analysis using Kupiec's test leads to similar results as the ones reached within our EVT analysis and further evidences that the CPT model is superior in matching realized returns.

\subsection{Prelec's weighting function parameter}

As another robustness check, we estimate the weighting function parameter $\omega$ of the RDEU model suggested by Prelec (1998) in order to test whether our conclusions are robust to other weighted functions formulations ${ }^{24}$. The Prelec weighting function $w_{p}^{+-}$is given by Eq. 17

$$
w_{p}^{+-}(p)=\exp \left(-(-\log (p))^{\omega}\right)
$$

where the parameter $\omega$ defines the curvature of the weighting function for both gains and losses, which also leads to $S$-shaped probability distortion functions. We note that according to Prelec (1998) the standard $\omega$ parameter value equals 0.65. Our time-varying and long-term (LT) estimates for $\omega$ are presented in Table 7, Panel A.

The long-term estimates of $\omega$ are somewhat in line with the one suggested by the RDEU but less so for the twelve-month horizon: $\omega$ estimated from the three-, six-, and twelve-months are $0.46,0.67$, and 1.11 , respectively. These parameters are somewhat consistent with our longterm estimates for $\gamma$ being, 0.75, 0.81, and 1.09 (see Table 1), as they suggest overweighting of small probabilities that fades with the increase in the option horizon. Similarly, timevarying estimates of $\omega$ also indicate more overweight of small probabilities than suggested by $\gamma$ estimations. We find the mean (0.95) and median (0.93) for time-varying estimates of $\omega$ from three-month options to be higher than the ones suggested by Prelec (1998). This outcome means that overweighting of small probabilities within the single stock option markets is less than suggested by RDEU (similar to our conclusion concerning CPT parameters) and that estimated Prelec parameters imply a less pronounced overweight of tails than suggested by our CPT parameter estimations. In line with our results for the CPT, for the six- and twelve-month maturities, underweight of small probabilities is, however, more frequent than an

\footnotetext{
${ }^{24} \mathrm{~A}$ major advance of Prelec's (1998) weighting function vis-à-vis the CPT is that it is monotonic for any value of $\omega$, whereas the CPT can have a non-monotonic probability weighting for low levels of $\gamma$.
} 
overweight. The average $\omega$ for the six-month options is 1.02 (median being 0.99), and for the twelve-months options is 1.05 (median being 1.07). The fact that investors tend to overweight small probabilities to a much lesser extent in the short-term and that estimates are higher than suggested by their respective lab-based estimates confirms our main findings.

[Please insert Table 7 about here]

The sample dependence observed in our main results is confirmed by the usage of Prelec's weighting function as overweight of tails is pervasive mostly in the 1998-2003 sample. Overall, the robustness checks following Prelec (1998) confirm our main findings regarding time-variation and sample dependence of overweighting of small probabilities, and reiterate our conclusion.

\subsection{Estimating time-varying $\gamma$ under different assumptions for $\delta, \alpha$ and $\beta$}

As an additional robustness test to our time-varying estimates of $\gamma$, we also run optimizations where we fix parameter $\delta$ instead of jointly optimizing it with $\gamma$. We impose $\delta=1$ (no overweight of small probabilities on the left-side of the distribution) or 0.69 , the value of $\delta$ within the CPT. In line with our previous robustness test, Table 7, Panels B and C, suggests that results from optimizations with different values for $\delta$ are qualitatively the same to our main results, i.e., a positive term structure and sample dependency of overweight of small probabilities. Unreported results also indicate a negative correlation between $\gamma$ and sentiment and high explanatory power of regressions. $R^{2}$ is between 13 and 21 percent for three- and six-month options and between 0 to 3 percent for twelve-month options. Though, neutral probability weighting on the left side of the distribution $(\delta=1)$ adjusts $\gamma$ downwards when compared to our main results. Conversely, when $\delta$ is 0.69 , an upwards adjustment to $\gamma$ estimates occurs.

Similarly, we also estimate $\gamma$ under different assumptions for $\alpha$ and $\beta$. We assumed $\alpha=\beta=1$ (no diminishing sensitivity to gains and losses) and $\alpha=\beta=0.75$ (more pronounced diminishing sensitivity to gains and losses) instead of the CPT parameterization $\alpha=\beta=0.88$. Our results, reported in Table 7, Panels D and E, suggest that lower sensitivity to gains and losses (higher $\alpha$ and $\beta$ ) leads to a decrease in overweight of small probabilities (higher $\gamma$ estimates), whereas higher sensitivity to gains and losses (lower $\alpha$ and $\beta$ ) leads to an increase in overweight of tails (lower $\gamma$ estimates). This effect is similar to the one observed by changes in $\lambda$ (described in Section 4.3), which also magnifies the sensitivity for losses when increased.

As indicated in Section 3.2, we have also estimated time-varying $\gamma$ using different lower (-0.25, 0 and 0.28) and upper bounds (1.2, 1.35, 1.5, 1.75 and 2). Results across bounds used differ to the extent that higher bounds produce upward shifts in the estimated $\gamma$ across all quantiles, median and averages to the extent that overweight of small probabilities becomes less pronounced but remain present. The time-variation pattern observed in Figure 3 and, more importantly, the strong negative relationship with sentiment reported in Table 4 are, though, extremely robust to changes in lower and upper optimization bounds. This result strengthens our conclusion that overweight of small probabilities is largely time varying and reflects investor 
sentiment.

\subsection{Overweight of (right) tails driven by IV of single stock options}

Finally, given that overweight of small probabilities by single stock call investors was most evident during the IT bubble period (as Table 3 suggests), we hereby evaluate whether this finding may have been driven by movements in the IV of index options rather than changes in the IV of single stock options. We perform such analysis because our methodology for calculation of average weighted stock IV volatilities partly relies on the IV on index options (as it depends on implied correlations), as Eqs. A.8i and A.8k in Appendix A.2 suggest. Essentially, we want to ensure that the overweight of small probabilities observed from our single stock options data is not caused by a rise in index options' IV. As overweight of small probabilities is a corollary of high IV skew ${ }^{25}$, we examine the IV skews (120 percent moneyness versus at-the-money, ATM) from both index options and from single stock options within our sample using a k-NearestNeighbors (KNN) algorithm (see Appendix A.4 for detail). Figure 4 depicts a scatter plot that relates single stock IV skews (on the y-axis) with index option IV skew (on the x-axis) overlaid with the decision boundary between overweight of tails (in red) and its absence (in blue), produced by the application of the KNN algorithm to our full data sample. The picture suggests that that overweight of small probabilities is almost never caused by positive index IV skews, whereas positive single stock IV skews very often produce overweight of tails rather than underweights. Overweight of tails are mostly caused by situation where single stock IV skew are higher than index IV skew, which suggest that either high single stock IV skews or low implied correlation are responsible for overweight of tails, not index options' IV. These conditions can be anecdotally confirmed by our observation of IV skews during the 2000's IT bubble. During that period, when overweight of tails was pervasive, IV skew from single options was quite high, close to +10 volatility points, whereas the same IV skew from index options reached extreme low levels such as -15 volatility points. This disconnect between the two IV markets, which drove the implied correlation to 2.8 percent (an extreme low level), suggests that the index options' IV was not the driver for overweight of tails during the IT bubble. These findings reiterate our suggestions that overweight of small probabilities observed in our sample is caused by trading in single stock options by retail investors, rather than activity in the index option market.

\section{Conclusion}

Single stock OTM call options are deemed overpriced because investors overpay for positively skewed securities, resembling lottery tickets. The CPT's probability weighting function of Tversky and Kahneman (1992) theoretical model provides an appealing explanation why these options are expensive: investors' preferences for positively skewed securities. In our empirical

\footnotetext{
${ }^{25}$ While this relation is widely acknowledged, Jarrow and Rudd (1982), Corrado and Su (1997) and Longstaff (1995) provide a formal theorem for the link between IV skew and risk-neutral.
} 
analysis, we find that the CPT subjective density function implied by single stock options outperforms the RND and two rational densities functions (from the power and exponential utilities) in matching the tails of realized equity returns. We estimate the CPT probability weighting function parameter $\gamma$ and find that they are qualitatively consistent with the one predicated by Tversky and Kahneman (1992), particularly for short-term options. This outcome endorses our hypothesis that investors in single stock call options are biased.

Our analysis provides detailed insights into the behavior of single stock option investors. Our empirical findings suggest that overweight of small probabilities is less pronounced than proposed by the CPT. We find the presence of a positive term structure of overweighting of tails, because it becomes less pronounced as the option maturity increases. Investors in single stock calls are more biased when trading short-term contracts, whereas they seem to be more rational (less biased) when trading long-term calls. This result is consistent with individual investors being the typical buyers of OTM single stock calls and the fact that they mostly use short-term options to speculate on the upside of equities.

We also find that investors overweighting of small probabilities is largely time-varying and sample dependent. Time-variation in $\gamma$ 's remains strong even when we account for different levels of loss aversion, different diminishing sensitivities to gains and losses, different degrees of overweighting of the left tail and an alternative (Prelec's) weighting function. The strong time-variation and sample dependency of suggest that investors do not have a static preference for skewness, but rather time-varying preferences or "bias in beliefs" (see Barberis, 2013).

Such time-variation in $\gamma$ is also confirmed by overweighting of tails to be pronounced in periods in which sentiment is high, for instance, the IT bubble period. This finding is consistent with the Baker and Wurgler (2007) sentiment measure being the main explanatory variable of overweighting of small probabilities. Our results challenge the view that single stock call options are structurally overpriced and offer the insight that overweight of tail events implied in these options are conditional on sentiment levels and option maturity rather than positive stock fundamentals, loss aversion levels or investor preferences for skewness.

Our findings have several important practical implications. First, the understanding of time-variation in investors' overweighting of small probabilities could be used in the development of behavioral option pricing models, which remains in its infancy . To the extent that overweighting of small probabilities is a latent variable or, simply, not trivial to estimate, we contemplate that future option pricing models should be more sentiment-aware than current ones. Second, of importance for such next generation option-pricing models is the inclusion of a positive term structure of tails' overweighting. Such potential modifications on options' pricing have large and direct consequences to risk-management, hedging and arbitrage activities. Third, from a financial stability point of view, investors' overweighting of small probabilities in single stock options could be of use to regulators for triangulating the presence of speculative equity markets bubbles. 


\section{A Appendix}

\section{A.1 Subject density function estimation}

We hereby present the derivations required to achieve Eq. (6) in the main text, Eq. (A.7) here, from Eq. (5), called here Eq. (A.1):

$$
\frac{w^{\prime}\left(F_{P}\left(S_{T}\right)\right) \cdot f_{P}\left(S_{T}\right)}{f_{Q}\left(S_{T}\right)}=\varsigma\left(S_{T}\right) .
$$

where $f_{P}\left(S_{T}\right)$ is the "real-world" probability distribution, $f_{Q}\left(S_{T}\right)$ is the RND, $\varsigma\left(S_{T}\right)$ is the pricing kernel, $w$ is the weighting function and $F_{P}\left(S_{T}\right)$ is the "real-world" cumulative density function.

The first step of our derivation entails re-arranging Eq. (A.1) into (A.2b) via Eq. (A.2a), which demonstrates that for the CPT to hold, the subjective density function should be consistent with the probability weighted EDF:

$$
\begin{gathered}
\underbrace{f_{Q}\left(S_{T}\right)}_{\text {RND }}=\underbrace{w^{\prime}\left(F_{P}\left(S_{T}\right)\right)}_{\text {probability weighing }} \cdot \underbrace{f_{P}\left(S_{T}\right)}_{\mathrm{EDF}} \cdot \underbrace{\varsigma\left(S_{T}\right)}_{\text {pricing kernel }} \\
\underbrace{f_{Q}\left(S_{T}\right)}_{\text {RND }}=\underbrace{f_{\widetilde{P}}\left(S_{T}\right)}_{\text {probability weighted EDF pricing kernel }} \\
\underbrace{\frac{f_{Q}\left(S_{T}\right)}{\lambda_{U^{\prime}\left(S_{T}\right)}^{\varsigma\left(S_{T}\right)}}}_{\text {Subjective density }}=\frac{f_{Q}\left(S_{T}\right)}{\varsigma\left(S_{T}\right)}=\underbrace{f_{\widetilde{P}}\left(S_{T}\right)}_{\text {probability weighted EDF }}
\end{gathered}
$$

Following Ait-Sahalia and Lo (2000) and Bliss and Panigirtzoglou (2004), Eq. (A.3) can be manipulated so that the time-preference constant $\Lambda$ of the pricing kernel vanishes, producing Eq. (A.4), which directly relates the probability weighted EDF, the RND, and the marginal utility, $U^{\prime}\left(S_{T}\right)$ :

$$
\underbrace{f_{\widetilde{P}}\left(S_{T}\right)}_{\text {probability weighted EDF }}=\underbrace{\frac{\lambda \frac{U^{\prime}\left(S_{T}\right)}{U^{\prime}\left(S_{t}\right)} Q\left(S_{T}\right)}{\int \frac{U^{\prime}\left(S_{t}\right)}{U^{\prime}(x)} Q(x) d x}=\frac{\frac{f_{Q}\left(S_{T}\right)}{U^{\prime}\left(S_{T}\right)}}{\int \frac{f_{Q}(x)}{U^{\prime}(x)} d x}}_{\text {Generic subjective density function }}
$$

where $\int \frac{Q(x)}{U^{\prime}(x)} d x$ normalizes the resulting subjective density function to integrate to one. Once the utility function is estimated, Eq. (A.4) allows us to convert RND into the probability weighted EDF. Eq. (A.4) can also be used to estimate the subjective density function for an (rational) investor that has power or exponential utility function, by disregarding the weighting function $W(\cdot)$, so the left-hand side of the equation becomes $f_{p}\left(S_{T}\right)$. In the remainder of the paper we call these subjective distributions power and exponential density functions. As we hypothesize that the representative investor has a CPT utility function, its marginal utility function is $U^{\prime}\left(S_{T}\right)=v^{\prime}\left(S_{T}\right)$, and, thus, $v^{\prime}\left(S_{T}\right)=\alpha S_{T}^{\alpha-1}$ for $S_{T}>=0$, and $v^{\prime}\left(S_{T}\right)=-\lambda \beta\left(-S_{T}\right)^{\beta-1}$ for $S_{T}<0$, leading to Eq. (A.5): 


$$
\begin{aligned}
& f_{\widetilde{P}}\left(S_{T}\right)=\frac{\frac{f_{Q}\left(S_{T}\right)}{\alpha S_{T}^{\alpha-1}}}{\int \frac{f_{Q}(x)}{\alpha x^{\alpha-1}} d x} \quad \text { for } \quad S_{T} \geq 0, \quad \text { and } \\
& \underbrace{f_{\widetilde{P}}\left(S_{T}\right)}_{\text {probability weighted EDF }}=\underbrace{\frac{\frac{f_{Q}\left(S_{T}\right)}{-\lambda \beta\left(-S_{T}\right)^{\beta-1}}}{\int \frac{f_{Q}(x)}{-\lambda \beta(-x)^{\beta-1}} d x}}_{\text {Partial CPT density function }} \text { for } S_{T}<0, \text { and }
\end{aligned}
$$

Eqs. (A.5) and (A.6), hence, relate the EDF where probabilities are weighted according to the CPT probability distortion functions, on the LHS, to the subjective density function derived from the CPT value function, on the RHS, separately for gains and losses, i.e., the PCPT density function. The relationships specified by Eqs. (A.5) and (A.6) fully state the relation we would like to depict, although one additional manipulation is convenient for our argumentation. Assuming that the function $w\left(F_{P}\left(S_{T}\right)\right)$ is strictly increasing over the domain $[0,1]$, there is a one-to-one relationship between $w\left(F_{P}\left(S_{T}\right)\right)$ and a unique inverse $w^{-1}\left(F_{P}\left(S_{T}\right)\right)$. So, result $f_{\widetilde{P}}\left(S_{T}\right)=w^{\prime}\left(F_{P}\left(S_{T}\right)\right) f_{P}\left(S_{T}\right)$ also implies $f_{\widetilde{P}}\left(S_{T}\right) \cdot\left(w^{-1}\right)^{\prime}\left(F_{P}\left(S_{T}\right)\right)=f_{P}\left(S_{T}\right)^{26}$. This outcome allows us to directly relate the original EDF to the CPT subjective density function, by "undoing" the effect of the CPT probability distortion functions within the PCPT density function:

$$
\underbrace{f_{P}\left(S_{T}\right)}_{\mathrm{EDF}}=\underbrace{\frac{\frac{f_{Q}\left(S_{T}\right)}{\nu^{\prime}\left(S_{T}\right)}}{\int \frac{f_{Q}(x)}{\nu^{\prime}(x)} d x}\left(w^{-1}\right)^{\prime}\left(F_{P}\left(S_{T}\right)\right)}_{\mathrm{CPT} \text { density function }}
$$

Thus, once the relation between the probability weighting function of EDF and the PCPT density is established, as in Eqs. (A.5) and (A.6), one can eliminate the weighting scheme affecting returns by applying the inverse of such weightings to the subjective density function without endangering such equalities, as in Eq. (A.7), numbered Eq. (6) in the main text.

\section{A.2 Single stock weighted average implied volatilities}

Starting from the portfolio variance formula, Eq. A.8a, we hereby provide the derivation of our single stock weighted-average implied volatility, given in Eq. A.8k:

$$
\sigma_{I}^{2}=\sum_{i, j=1}^{n} w_{i} w_{j} \rho_{i j} \sigma_{i} \sigma_{j}
$$

where,

$$
\rho_{i j}(x)=\left\{\begin{array}{lll}
\bar{\rho}, & \text { if } \quad i \neq j \\
1, & \text { if } \quad i=j
\end{array}\right.
$$

\footnotetext{
${ }^{26} \mathrm{~A}$ drawback of the CPT model is that it allows for non-strictly increasing functions, which would not allow invertibility. This is the reason why the newer literature on probability distortions functions favors other strictly monotonic functions, such as Prelec's $(1998) w(p)=e^{-(-\ln (p))^{\delta}}$, as the weighting functions. Nevertheless, because the CPT parameters of our interest $(\gamma=0.61 ; \delta=0.69)$ impose strict monotonicity, we can obtain the inverse of the probability function, $w^{-1}(p)$ numerically.
} 
and where $\sigma_{I}^{2}$ is the equity index option implied variance and $i$ and $j$ are indexes for the constituents of such equity index, then:

$$
\begin{aligned}
& \sigma_{I}^{2}=\bar{\rho} \sum_{i \neq j}^{n} w_{i} w_{j} \rho_{i j} \sigma_{i} \sigma_{j}+\sum_{i=1}^{n} w_{i}^{2} \sigma_{i}^{2}, \\
= & \bar{\rho} \sum_{i, j=1}^{n} w_{i} w_{j} \rho_{i j} \sigma_{i} \sigma_{j}+(1-\bar{\rho}) \sum_{i=1}^{n} w_{i}^{2} \sigma_{i}^{2}, \\
= & \bar{\rho}\left(\sum_{i=1}^{n} w_{i} \sigma_{i}\right)^{2}+(1-\bar{\rho}) \sum_{i=1}^{n} w_{i}^{2} \sigma_{i}^{2}, \\
= & \left(\sum_{i=1}^{n} w_{i} \sigma_{i}\right)^{2}+\sum_{i=1}^{n} w_{i}^{2} \sigma_{i}^{2}-\bar{\rho} \sum_{i=1}^{n} w_{i}^{2} \sigma_{i}^{2}, \\
=\bar{\rho}( & \left.\left(\sum_{i=1}^{n} w_{i} \sigma_{i}\right)^{2}-\sum_{i=1}^{n} w_{i}^{2} \sigma_{i}^{2}\right)+\sum_{i=1}^{n} w_{i}^{2} \sigma_{i}^{2}, \\
& \bar{\rho} \approx \frac{\sigma_{I}^{2}-\sum_{i, j=1}^{n} w_{i}^{2} \sigma_{i}^{2}}{\left(\sum_{i=1}^{n} w_{i} \sigma_{i}\right)^{2}-\sum_{i, j=1}^{n} w_{i}^{2} \sigma_{i}^{2}} .
\end{aligned}
$$

As $\sum_{i, j=1}^{n} w_{i}^{2} \sigma_{i}^{2}$ is relatively small, we can simplify A.8h into A.8i, the implied correlation:

$$
\bar{\rho} \approx \frac{\sigma_{I}^{2}}{\left(\sum_{i=1}^{n} w_{i} \sigma_{i}\right)^{2}}
$$

To obtain the single stock weighted average implied volatility (Eq. A.8k, we then square root both sides of the approximation and re-arrange its terms:

$$
\begin{gathered}
\sqrt{\bar{\rho}} \approx \frac{\sigma_{I}}{\left(\sum_{i=1}^{n} w_{i} \sigma_{i}\right)} \\
\sum_{i=1}^{n} w_{i} \sigma_{i} \approx \frac{\sigma_{I}}{\sqrt{\bar{\rho}}}
\end{gathered}
$$

\section{A.3 Least Absolute Shrinkage and Selection Operator (Lasso)}

The regression coefficients obtained by the Lasso methodology applied $\left(\beta_{\theta}^{L}\right)$ are estimated by minimizing the quantity:

$$
\sum_{i=1}^{n}\left(y_{1}-\beta_{0}-\sum_{j=1}^{p} \beta_{j} x_{i j}\right)^{2}+\kappa \sum_{j=1}^{p}\left|\beta_{j}\right|=R S S+\kappa \sum_{j=1}^{p}\left|\beta_{j}\right|
$$

where $\kappa$ is the tuning parameter, which is estimated via cross-validation. The cross-validation applied by us uses ten equal-size splits of our overall data set. 


\section{A.4 k-Nearest-Neighbor classifier}

The k-Nearest-Neighbor (KNN) classifier is one of the approaches in machine learning that attempts to estimate the conditional distribution of the explained variable $(Y)$ given the explanatory variables $(X)$ and, subsequently, classify new observations to the class with highest estimated probability. The KNN classifier uses the Euclidean distance to first identify the closest $\mathrm{k}^{\text {th }}$ observations within the training data (in-sample data) to a new test (out-of-sample) observation provided $\left(x_{0}\right)$. Such neighborhood of points around the test observation $x_{0}$ is defined as $\mathbb{N}_{0}$. KNN, then, estimates the conditional probability of $x_{0}$ to belong to a class $j$ as the percentage of old observations $\left(y_{i}\right)$ in the neighborhood $\mathbb{N}_{0}$ whose class is also $j$ :

$$
\operatorname{Pr}\left(Y=j \mid X=x_{0}\right)=\frac{1}{k} \sum_{i \in \mathbb{N}_{0}} I\left(y_{i}=j\right)
$$

In a third step, KNN applies the Bayes rule to perform out-of-sample classification (in test data) of $x_{0}$ to the class with the largest probability. For further details, see Hastie et al. (2008). 


\section{B Appendix}

\section{B.1 Welch and Goyal (2008) equity market predictors}

The complete set and summarized descriptions of variables provided by Welch and Goyal $(2008)^{27}$ that are used in our study is given as:

1. Dividendprice ratio (log), D/P: Difference between the log of dividends paid on the S\&P 500 index and the log of stock prices (S\&P 500 index).

2. Dividend yield (log), D/Y: Difference between the log of dividends and the $\log$ of lagged stock prices.

3. Earnings, E12: 12-month moving sum of earnings on teh S\&P500 index.

4. Earnings-price ratio (log), E/P: Difference between the log of earnings on the S\&P 500 index and the log of stock prices.

5. Dividend-payout ratio (log), D/E: Difference between the log of dividends and the log of earnings.

6. Stock variance, SVAR: Sum of squared daily returns on the S\&P 500 index.

7. Book-to-market ratio, B/M: Ratio of book value to market value for the Dow Jones Industrial Average.

8. Net equity expansion, NTIS: Ratio of twelve-month moving sums of net issues by NYSE-listed stocks to total end-of-year market capitalization of NYSE stocks.

9. Treasury bill rate, TBL: Interest rate on a three-month Treasury bill.

10. Long-term yield, LTY: Long-term government bond yield.

11. Long-term return, LTR: Return on long-term government bonds.

12. Term spread, TMS: Difference between the long-term yield and the Treasury bill rate.

13. Default yield spread, DFY: Difference between BAA- and AAA-rated corporate bond yields.

14. Default return spread, DFR: Difference between returns of long-term corporate and government bonds.

15. Cross-sectional premium, CSP: measures the relative valuation of high- and low-beta stocks.

16. Inflation, INFL: Calculated from the CPI (all urban consumers) using $t-1$ information due to the publication lag of inflation numbers.

${ }^{27}$ Available at http://www.hec.unil.ch/agoyal/. 


\section{References}

Ait-Sahalia, Y., Lo, A., 2000. Nonparametric risk management and implied risk aversion. Journal of Econometrics 94 (3), 90-51.

Anagnou, I., Bedendo, M., Hodges, S., Tompkins, R., 2002. The relationship between implied and realised probability density function. Working paper, University of Warwick and the University of Technology, Vienna.

Baker, M., Wurgler, J., 2007. Investor sentiment in the stock market. Journal of Economic Perspectives 21 (Spring), 129-157.

Barber, B., Odean, T., 2008. All that glitters: The effect of attention and news on the buying behavior of individual and institutional investors. Review of Financial Studies 21, 785-818.

Barberis, N., 2013. The psychology of tail events: Progress and challenges. American Economic Review 103 (3), 611-616.

Barberis, N., Huang, M., 2001. Mental accounting, loss aversion and individual stock returns. Journal of Finance 56 (4), 1247-1292.

Barberis, N., Huang, M., 2008. Stocks as lotteries: The implication of probability weighting for security prices. American Economic Review 98 (5), 2066-2100.

Barberis, N., Huang, M., Santos, T., 2001. Prospect theory and asset prices. Quarterly Journal of Economics 56 (1), 1-53.

Barberis, N., Shleifer, A., Vishny, R., 1998. A model of investor sentiment. Journal of Financial Economics 49 (3), 307-343.

Bates, D., 2003. Empirical option pricing: A retrospection. Journal of Econometrics 116, 387404.

Bauer, R., Cosemans, M., Eichholtz, P., 2009. Option trading and individual investor performance. Journal of Banking ES Finance 33 (4), 731-746.

Benartzi, S., Thaler, R., 1995. Myopic loss aversion and the equity premium puzzle. Quarterly Journal of Economics 110 (1), 73-92.

Berger, D., Turtle, H., 2015. Sentiment bubbles. Journal of Financial Markets 23 (March), 59-74.

Bliss, R., Panigirtzoglou, N., 2004. Option-implied risk aversion estimates. Journal of Finance 59 (1), 407-446.

Blume, M., Keim, D., 2012. Institutional investors and stock market liquidity: Trends and relationships. Working paper: The Wharton School.

Bollen, N., Whaley, R., 2004. Does net buying pressure affect the shape of implied volatility function? Journal of Finance 59 (2), 711-754.

Bollerslev, T., Tauchen, G., Zhou, H., 2009. Exptected stock returns and variance risk premia. Review of Financial Studies 22 (11), 4463-4492.

Boyer, B., Vorkink, K., 2014. Stock options as lotteries. Journal of Finance 69 (4), 1484-1527. 
Chabi-Yo, F., Song, Z., 2013. Recovering the probability weights of tail events with volatility risk from option prices. Working paper Ohio State University and Federal Reserve System, $1-55$.

Chen, Y., Kumar, A., Zhang, C., 2015. Searching for gambles: investor attention, gambling sentiment, and stock market outcomes. SSRN working paper 2635572.

Choy, S., 2015. Retail clientele and option returns. Journal of Banking \& Finance 51 (5), 141-159.

Conrad, J., Dittmar, R., Ghysels, E., 2013. Ex-ante skewness and expected stock returns. Journal of Finance 68 (1), 85-124.

Corrado, C., Su, T., 1997. Implied volatility skews and stock index skewness and kurtosis implied by s\&p 500 index option prices. Journal of Derivatives 4 (4), 8-19.

Danielsson, J., Jorgensen, B., Sarma, M., de Vries, C., 2006. Comparing downside risk measures for heavy tailed distributions. Economics Letters 92 (2), 202-208.

De Haan, L., Jansen, D., Koedijk, K., de Vries, C., 1994. Safety first portfolio selection, extreme value theory and long run asset risks. In Proceedings from a Conference on Extreme Value Theory and Applications, Galambos J (ed.), Kluwer Academic: Boston, MA,, 471-487.

Devroye, L., 1986. Non-uniform random variate generation. Springer-Verlag, New York.

Dierkes, M., 2009. Option-implied risk attitude under rank-dependent utility. Unpublished working paper. University of Münster, Münster, Germany.

Felix, L., Kraussl, R., Stork, P., 2016. The 2011 european short sable ban: A cure or a curse? Journal of Financial Stability 25, 115-131.

Figlewski, S., 2010. Estimating the implied risk neutral density for the u.s. market portfolio. In Volatility and Time Series Econometrics: Essays in Honor of Robert F. Engle - Oxford University Press.

Fox, C., Rogers, B., Tversky, A., 1996. Options traders exhibit subadditive decision weights. Journal of Risk and Uncertainty 13, 5-17.

Frazzini, A., Pedersen, L., 2014. Betting against beta. Journal of Financial Economics 111 (1), $1-25$.

Garleanu, N., Pedersen, L. H., Poteshman, A. M., 2009. Demand-based option pricing. Review of Financial Studies 22 (10), 4259-4299.

Green, T., Hwang, B.-H., 2011. Initial public offering as lotteries: skewness preferences and first-day returns. Management Science 86 (2), 432-444.

Han, B., 2008. Investor sentiment and option prices. Review of Financial Studies 21 (1), 387414.

Hastie, T., Tibshirani, R., Friedman, J., 2008. The elements of statistical learning: data mining, inference, and prediction (2nd ed.), Springer-Verlag.

Hill, B., 1975. A simple general approach to inference about the tail of a distribution. Annals of Statistics 3 (5), 1163-1173. 
Hsu, M., Krajbich, I., Zhao, C., Camerer, C., 2009. Neural response to reward anticipation under risk is nonlinear in probabilities. Journal of Neurosciencel 29 (7), 2231-2237.

Ilmanen, A., 2012. Do financial markets reward buying or selling insurance and lottery tickets? Financial Analyst Journal 68 (5), 26-36.

Jackwerth, J., 2000. Recovering risk aversion from option prices and realized returns. Review of Financial Studies 13 (2), 433-451.

Jarrow, R., Rudd, A., 1982. , approximate option valuation for arbitrary stochastic processes. Journal of Financial Economics 10 (5), 347-369.

Jiao, Y., 2016. Lottery preference and earnings announcement premia. SSRN Working Paper 2522798 .

Kahneman, D., Tversky, A., 1979. Prospect theory: Na analysis of decision under risk. Journal of Financial Economics 47 (2), 263-291.

Kang, B., Kim, T., 2006. Option-implied risk preferences: An extension to wider classes of utility functions. Journal of Financial Markets 9 (2), 180-198.

Kliger, D., Levy, O., 2009. Theories of choice under risk: Insights from financial markets. Journal of Economic Behavior $\mathscr{G}$ Organization 71 (2), 330-346.

Kumar, A., 2009. Who gambles in the stock market? Journal of Finance 64 (4), 1889-1933.

Kupiec, P., 1995. Techniques for verifying the accuracy of risk management models. Journal of Derivatives 3, 73-84.

Lakonishok, J., Lee, I., Pearson, N., Poteshman, A., 2007. Option market activity. Review of Financial Studies 20 (3), 813-857.

Longstaff, F., 1995. Option pricing and the martingale restriction. Review of Financial Studies 8 (4), 1091-1124.

Mitton, T., Vorkink, K., 2007. Equilibrium underdiversification and the preference for skewness. Review of Financial Studies 20 (4), 1255-1288.

Nelson, D., 1991. Conditional heteroskedasticity in asset returns: A new approach. Econometrica 59 (2), 347-370.

Polkovnichenko, V., Zhao, F., 2013. Probability weighting functions implied in option prices. Journal of Financial Economics 107 (3), 580-609.

Prelec, D., 1998. The probability weighting function. Econometrica 66 (3), 497-527.

Rosenberg, J., Engle, R., 2002. Empirical pricing kernels. Journal of Financial Economics $64(3), 341-372$.

Straetmans, S., Verschoor, W., Wolff, C., 2008. Extreme us stock market fluctuations in the the wake of 9/11. Journal of Applied Econometrics 23 (1), 17-42.

Tibshirani, R., 1996. Regression shrinkage and selection via the lasso. Journal of the Royal Statistical Society 58 (1), 267-288.

Tversky, A., Kahneman, D., 1992. Advances in prospect theory: Cumulative representation of uncertainty. Journal of Risk and Uncertainty 5 (4), 297-323. 
Vilkov, G., Xiao, Y., 2013. Option-implied information and predictability of extreme returns. SAFE (Goethe University Frankfurt) Working Paper Series 5, 1-36.

von Neumann, J., Morgenstern, O., 1947. Theory of games and economic behavior, 2nd edition. Princeton University Press.

Welch, I., Goyal, A., 2008. A comprehensive look at the empirical performance of equity premium prediction. Review of Financial Studies 21 (4), 1455-1508.

Wu, G., Gonzalez, R., 1996. Curvature of the probability weighting function. Management Science 42 (12), 1676-1690. 


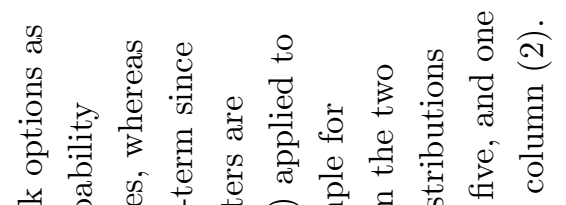

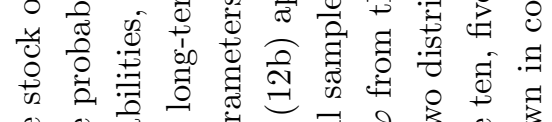

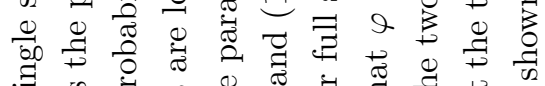
ज诸

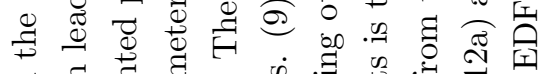

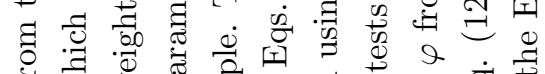

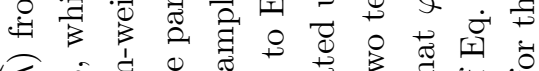

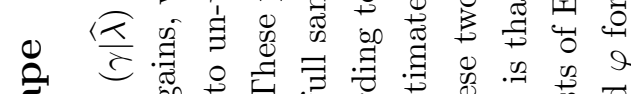

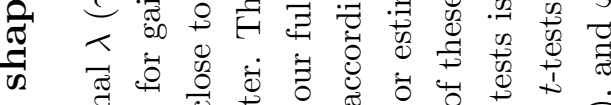
ส

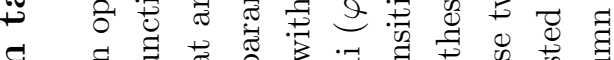

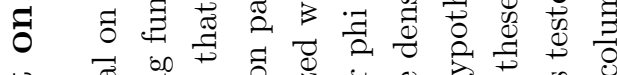

崩

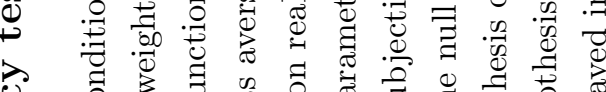
仓

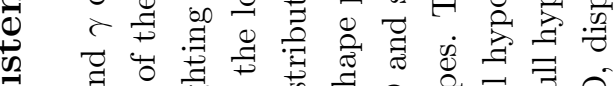
至 ठ ర

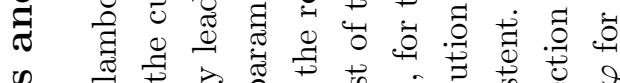
o

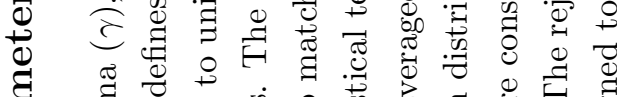

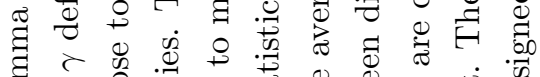

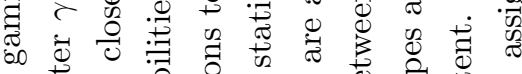

ప.

F

O

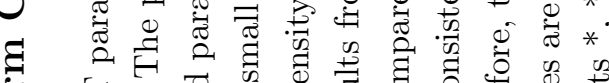

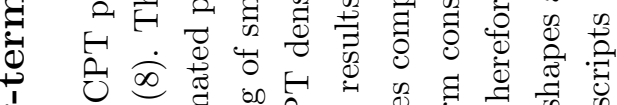

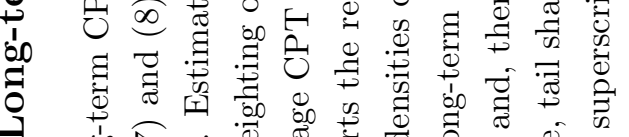
-

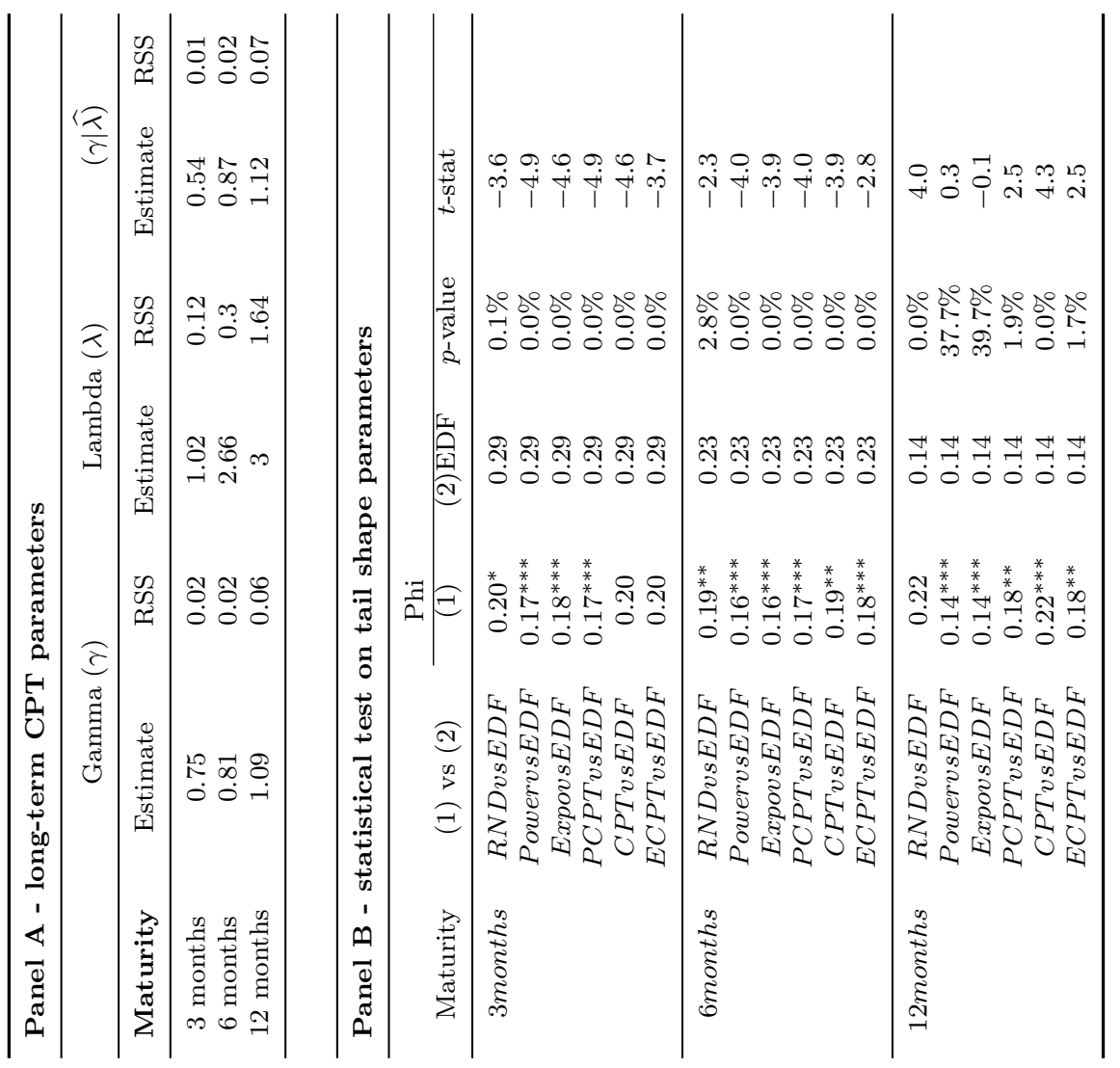

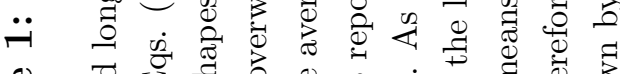

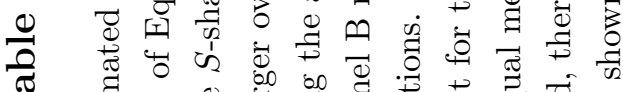

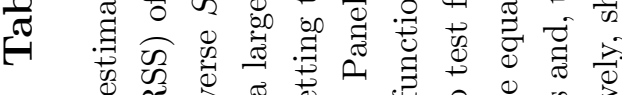

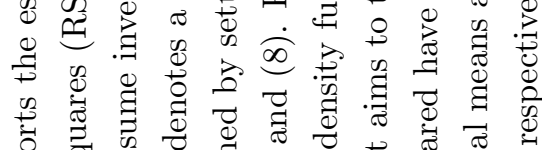
范 过

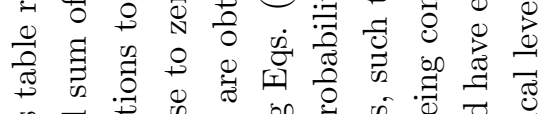

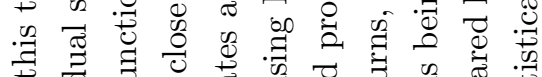

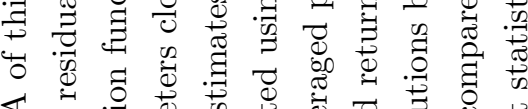
《

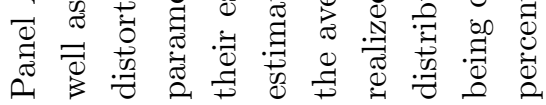




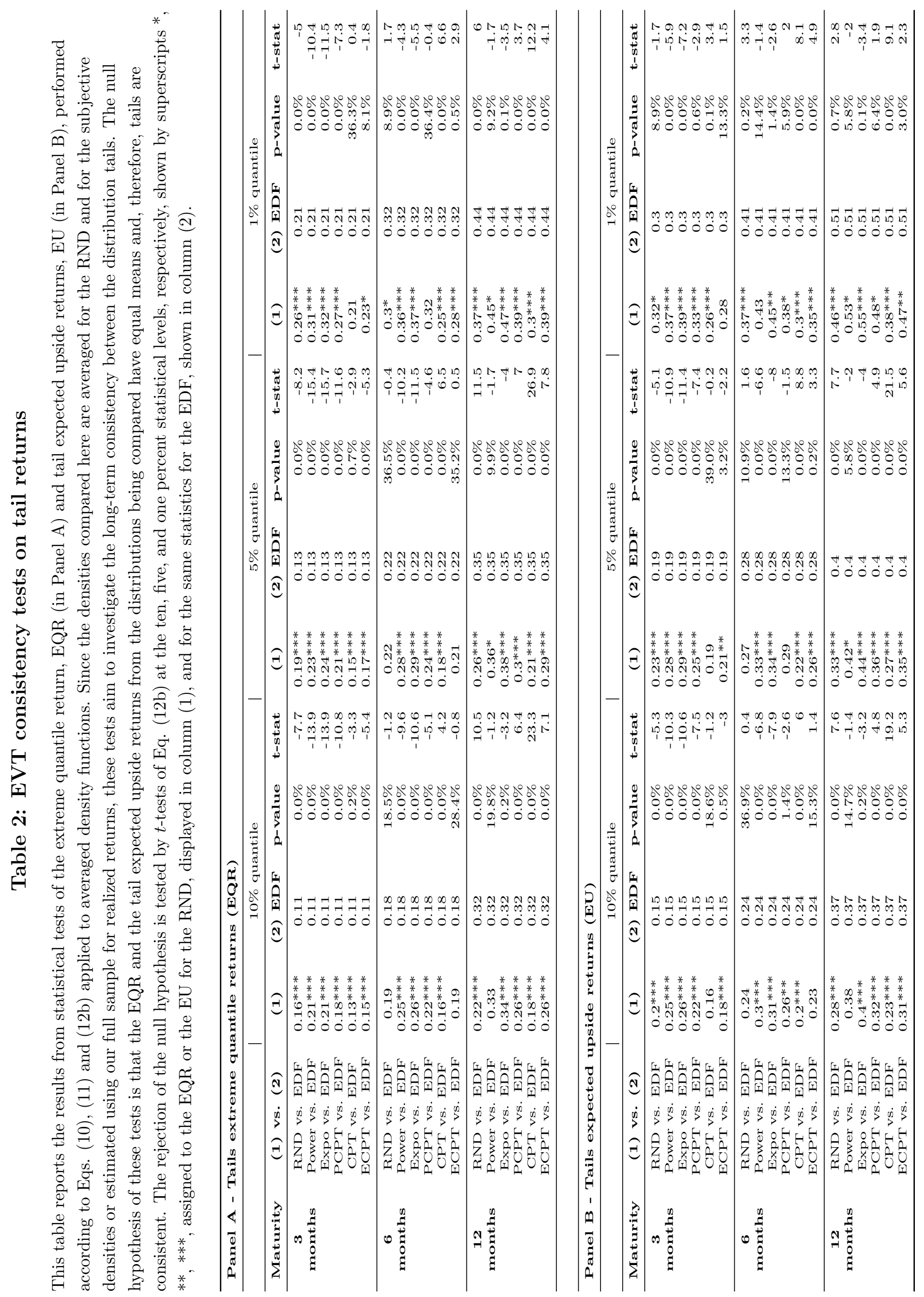




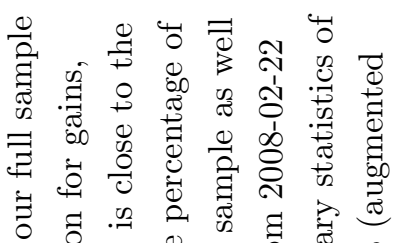

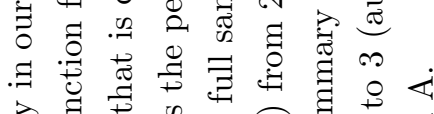

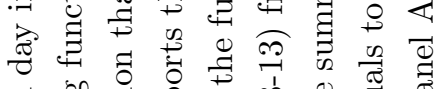

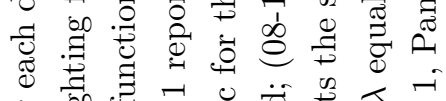

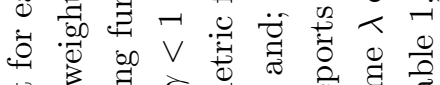

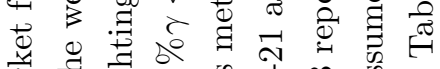
‡ँच

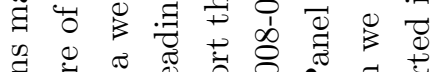
告

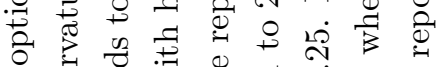

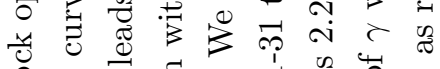

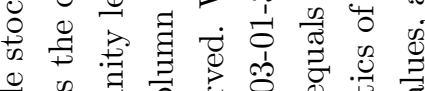

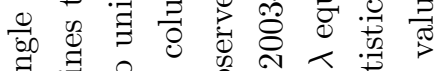

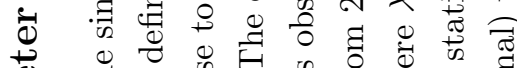

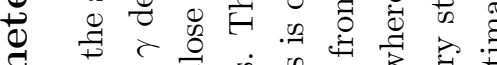
ส व

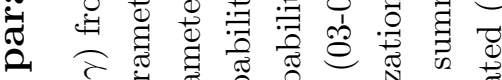

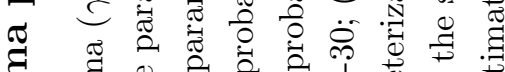

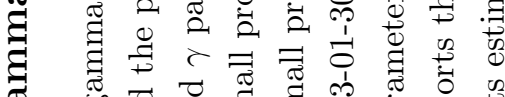
ब.

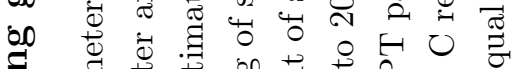

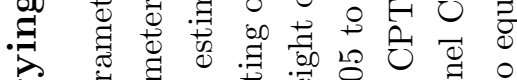

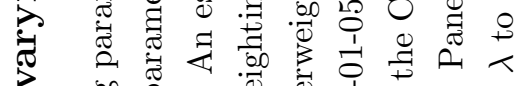

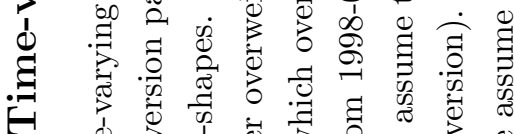

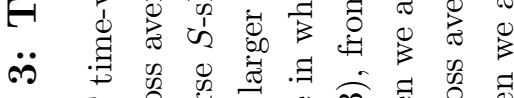

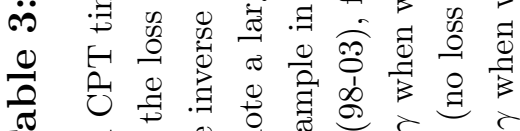

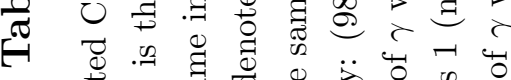

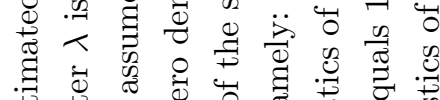

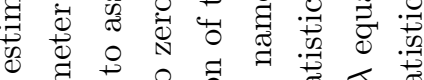

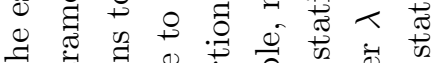

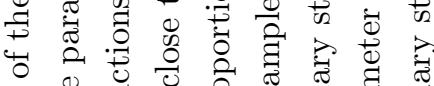

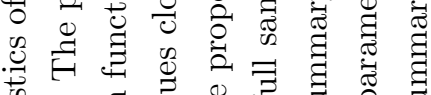

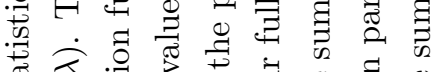

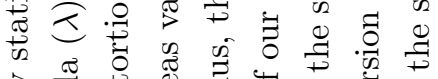

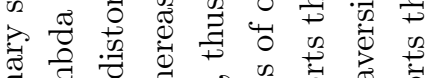

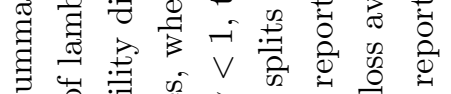
के

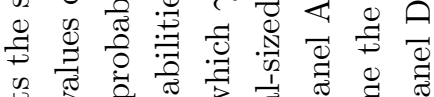

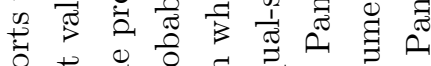

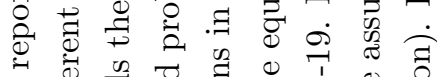

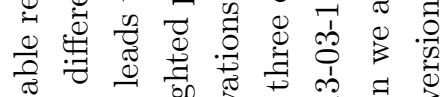

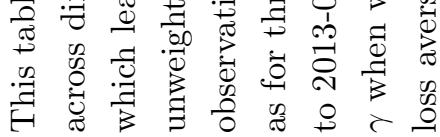

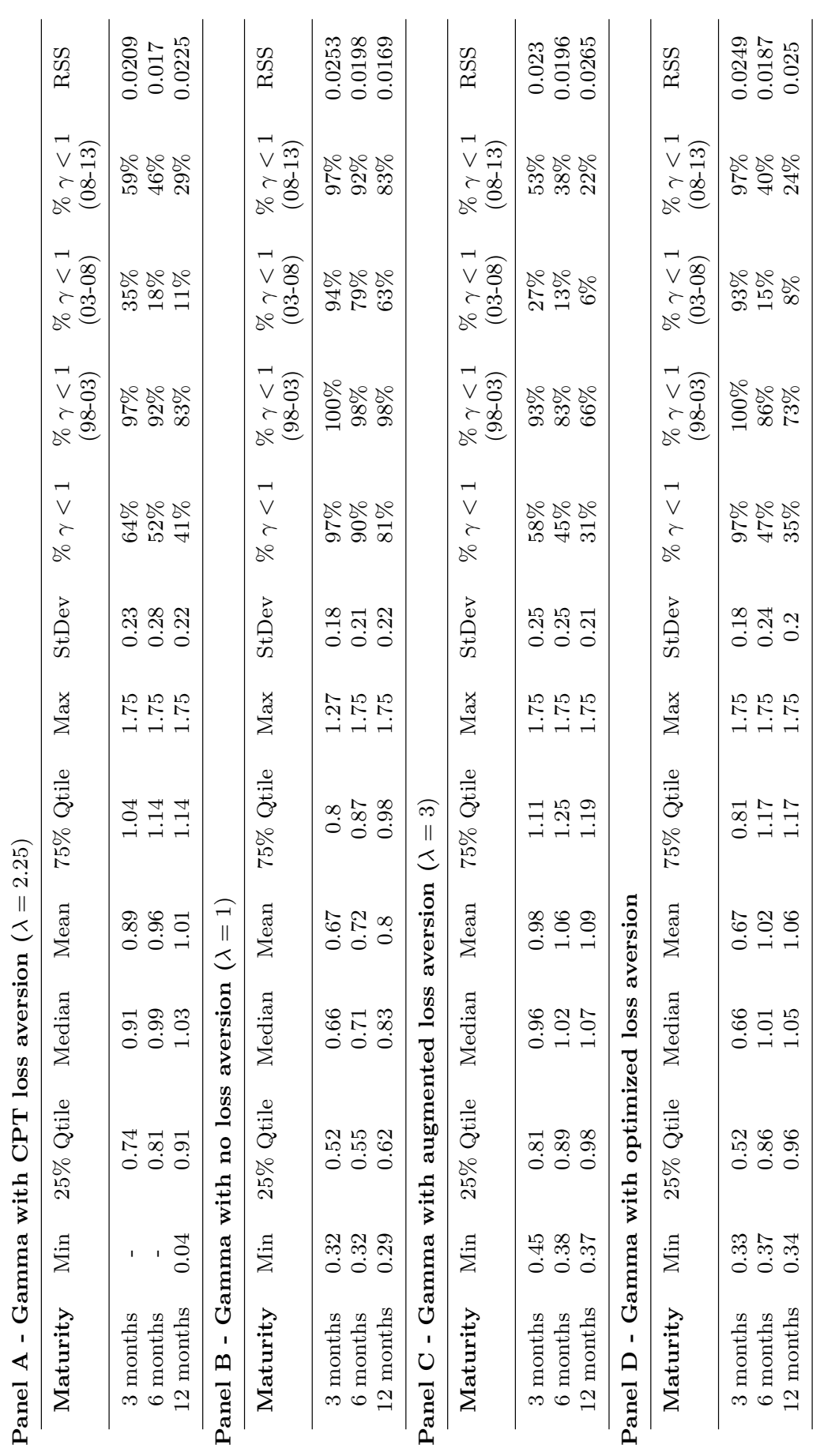




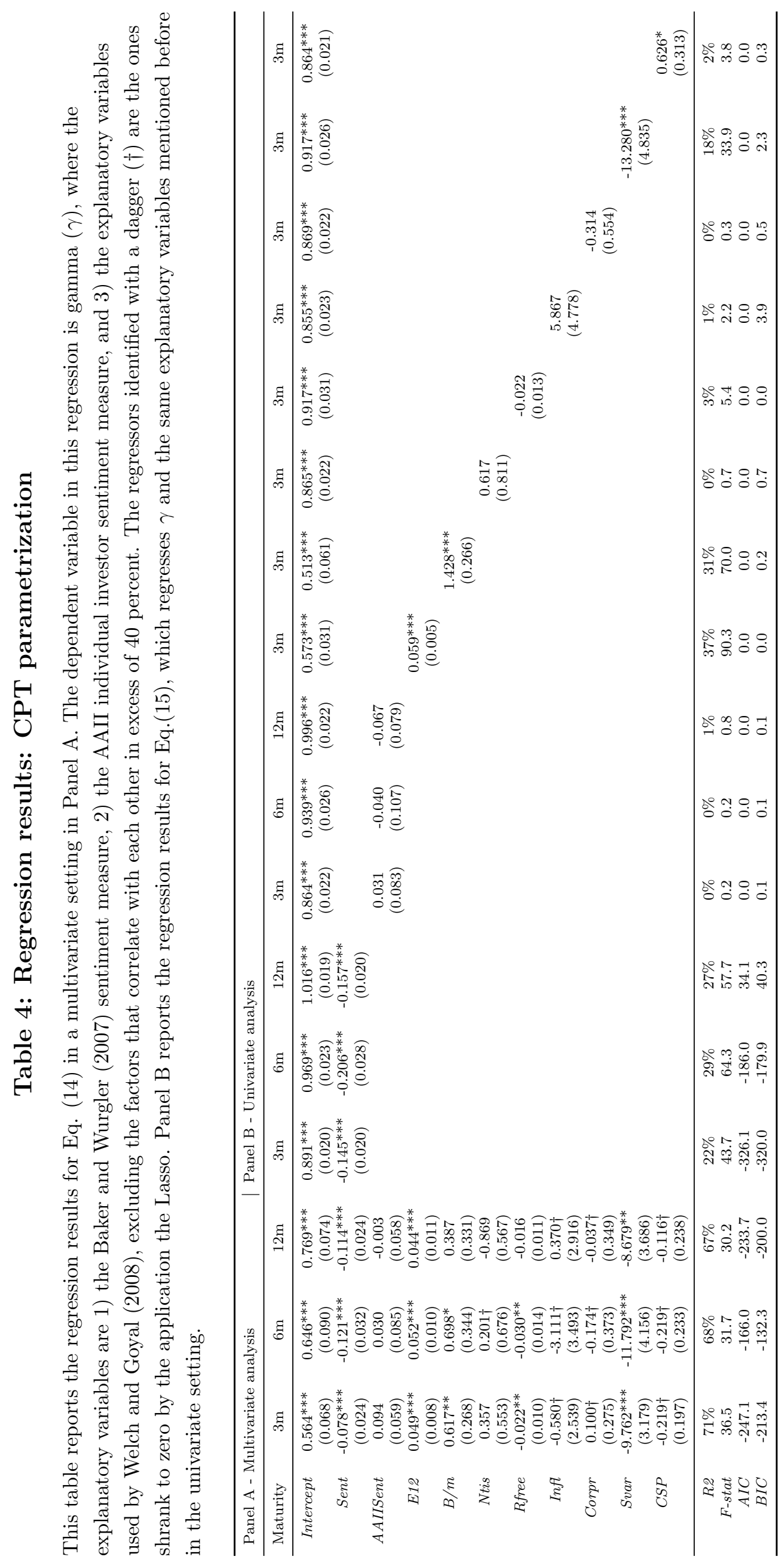




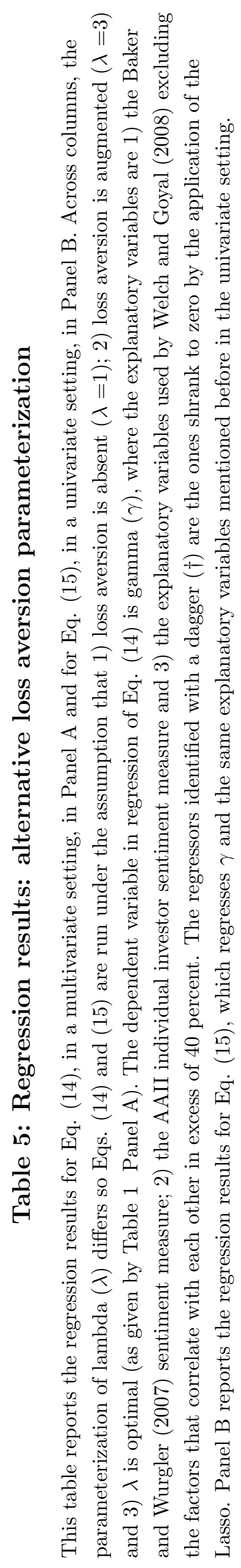

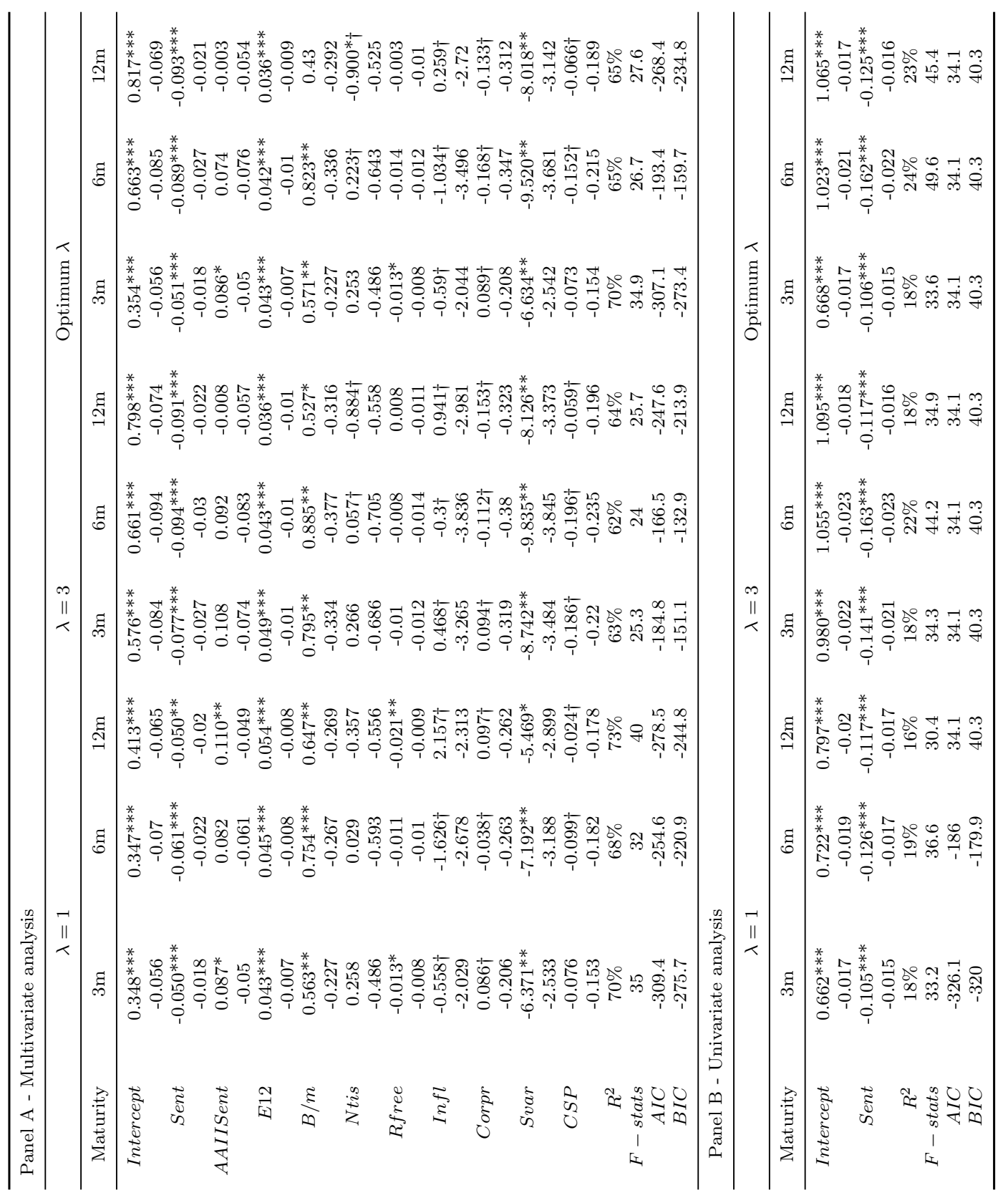




\section{Table 6: Robustness checks: Kupiec's test}

This table reports the results from Kupiec's (1995) percentage of failure (POF) test for violations of the extreme quantile returns (EQR) from the empirical density function (EDF) by the EQR of a set of RND and subjective density functions. The test is performed as a robustness check to the extreme value theory (EVT)-based tests performed on the EQR and on the expected upside returns. The null hypothesis, which is designed as a log-likelihood ratio test (Eq. (16)), is that the realized probability of failure $\left(\frac{v}{n}\right)$ matches the predicted one $p^{*}$. Thus if the $L R$ exceeds the critical value, $\chi^{2}(1)=3.841$, such a hypothesis is rejected at the five percent level. Translating the methodology to our empirical problem, $\left(p^{*}\right)$ becomes the assumed probability that the EQR of the subjective and of the risk-neutral densities will violate the EQR of the realized returns, where $\frac{v}{n}$ is the realized number of violations. We note that because we apply Kupiecs test to the upside returns, violations mean that returns are higher than a positive threshold.

\begin{tabular}{|c|c|c|c|c|c|c|c|c|c|}
\hline \multicolumn{10}{|c|}{ Panel A - Three-month calls } \\
\hline & \multicolumn{3}{|c|}{ EQR $90 \%$} & \multicolumn{3}{|c|}{ EQR $95 \%$} & \multicolumn{3}{|c|}{ EQR $99 \%$} \\
\hline $\mathrm{p}=10 \%$ & $\mathrm{POF}$ & p-value & LR-stat & POF & p-value & LR-stat & $\mathrm{POF}$ & p-value & LR-stat \\
\hline RND vs EDF & $99.9 \%$ & 0.0000 & $\infty$ & $99.2 \%$ & 0.0000 & $\infty$ & $50.5 \%$ & 0.0000 & 414.8 \\
\hline Power vs EDF & $100.0 \%$ & 0.0000 & $\infty$ & $100.0 \%$ & 0.0000 & $\infty$ & $84.7 \%$ & 0.0000 & $\infty$ \\
\hline Expo vs EDF & $100.0 \%$ & 0.0000 & $\infty$ & $100.0 \%$ & 0.0000 & $\infty$ & $86.8 \%$ & 0.0000 & $\infty$ \\
\hline PCPT vs EDF & $100.0 \%$ & 0.0000 & $\infty$ & $100.0 \%$ & 0.0000 & $\infty$ & $67.2 \%$ & 0.0000 & 752.0 \\
\hline CPT vs EDF & $58.2 \%$ & 0.0000 & 559.6 & $45.7 \%$ & 0.0000 & 333.3 & $16.0 \%$ & 0.0002 & 13.6 \\
\hline $\mathrm{p}=5 \%$ & $\mathrm{POF}$ & p-value & LR-stat & $\mathrm{POF}$ & p-value & LR-stat & $\mathrm{POF}$ & p-value & LR-stat \\
\hline RND vs EDF & $99.9 \%$ & 0.0000 & $\infty$ & $99.2 \%$ & 0.0000 & $\infty$ & $50.5 \%$ & 0.0000 & 671.5 \\
\hline Power vs EDF & $100.0 \%$ & 0.0000 & $\infty$ & $100.0 \%$ & 0.0000 & $\infty$ & $84.7 \%$ & 0.0000 & $\infty$ \\
\hline Expo vs EDF & $100.0 \%$ & 0.0000 & $\infty$ & $100.0 \%$ & 0.0000 & $\infty$ & $86.8 \%$ & 0.0000 & $\infty$ \\
\hline PCPT vs EDF & $100.0 \%$ & 0.0000 & $\infty$ & $100.0 \%$ & 0.0000 & $\infty$ & $67.2 \%$ & 0.0000 & $\infty$ \\
\hline CPT vs EDF & $58.2 \%$ & 0.0000 & 861.9 & $45.7 \%$ & 0.0000 & 561.3 & $16.0 \%$ & 0.0000 & 65.5 \\
\hline \multicolumn{10}{|c|}{ Panel B - Six-month calls } \\
\hline & \multicolumn{3}{|c|}{ EQR $90 \%$} & \multicolumn{3}{|c|}{ EQR $95 \%$} & \multicolumn{3}{|c|}{ EQR $99 \%$} \\
\hline $\mathrm{p}=10 \%$ & $\mathrm{POF}$ & p-value & LR-stat & POF & p-value & LR-stat & POF & p-value & LR-stat \\
\hline RND vs EDF & $99.9 \%$ & 0.0000 & $\infty$ & $93.3 \%$ & 0.0000 & $\infty$ & $13.8 \%$ & 0.0160 & 5.8 \\
\hline Power vs EDF & $99.9 \%$ & 0.0000 & $\infty$ & $97.7 \%$ & 0.0000 & $\infty$ & $22.1 \%$ & 0.0000 & 49.7 \\
\hline Expo vs EDF & $99.9 \%$ & 0.0000 & $\infty$ & $97.8 \%$ & 0.0000 & $\infty$ & $23.0 \%$ & 0.0000 & 56.4 \\
\hline PCPT vs EDF & $99.9 \%$ & 0.0000 & $\infty$ & $97.3 \%$ & 0.0000 & $\infty$ & $17.0 \%$ & 0.0000 & 18.2 \\
\hline CPT vs EDF & $62.4 \%$ & 0.0000 & 647.0 & $36.3 \%$ & 0.0000 & 197.3 & $5.7 \%$ & 0.0019 & 9.6 \\
\hline $\mathrm{p}=5 \%$ & $\mathrm{POF}$ & p-value & LR-stat & $\mathrm{POF}$ & p-value & LR-stat & $\mathrm{POF}$ & p-value & LR-stat \\
\hline RND vs EDF & $99.9 \%$ & 0.0000 & $\infty$ & $93.3 \%$ & 0.0000 & $\infty$ & $13.8 \%$ & 0.0000 & 44.8 \\
\hline Power vs EDF & $99.9 \%$ & 0.0000 & $\infty$ & $97.7 \%$ & 0.0000 & $\infty$ & $22.1 \%$ & 0.0000 & 137.7 \\
\hline Expo vs EDF & $99.9 \%$ & 0.0000 & $\infty$ & $97.8 \%$ & 0.0000 & $\infty$ & $23.0 \%$ & 0.0000 & 149.7 \\
\hline PCPT vs EDF & $99.9 \%$ & 0.0000 & $\infty$ & $97.3 \%$ & 0.0000 & $\infty$ & $17.0 \%$ & 0.0000 & 76.0 \\
\hline CPT vs EDF & $62.4 \%$ & 0.0000 & $\infty$ & $36.3 \%$ & 0.0000 & 369.9 & $5.7 \%$ & 0.5474 & 0.4 \\
\hline \multicolumn{10}{|c|}{ Panel C - Twleve-month calls } \\
\hline & \multicolumn{3}{|c|}{ EQR $90 \%$} & \multicolumn{3}{|c|}{ EQR $95 \%$} & \multicolumn{3}{|c|}{ EQR $99 \%$} \\
\hline $\mathrm{p}=10 \%$ & $\mathrm{POF}$ & $\mathrm{p}$-value & LR-stat & $\mathrm{POF}$ & p-value & LR-stat & $\mathrm{POF}$ & p-value & LR-stat \\
\hline RND vs EDF & $62.8 \%$ & 0.0000 & 655.1 & $25.0 \%$ & 0.0000 & 72.9 & $20.3 \%$ & 0.0000 & 37.0 \\
\hline Power vs EDF & $93.5 \%$ & 0.0000 & $\infty$ & $42.5 \%$ & 0.0000 & 283.5 & $29.3 \%$ & 0.0000 & 114.7 \\
\hline Expo vs EDF & $94.6 \%$ & 0.0000 & $\infty$ & $43.1 \%$ & 0.0000 & 292.7 & $30.4 \%$ & 0.0000 & 126.2 \\
\hline PCPT vs EDF & $79.5 \%$ & 0.0000 & 1067.2 & $36.1 \%$ & 0.0000 & 194.7 & $24.4 \%$ & 0.0000 & 68.3 \\
\hline CPT vs EDF & $29.4 \%$ & 0.0000 & 115.2 & $7.2 \%$ & 0.0480 & 3.9 & $8.4 \%$ & 0.2666 & 1.2 \\
\hline $\mathrm{p}=5 \%$ & $\mathrm{POF}$ & $\mathrm{p}$-value & LR-stat & $\mathrm{POF}$ & p-value & LR-stat & $\mathrm{POF}$ & p-value & LR-stat \\
\hline RND vs EDF & $62.8 \%$ & 0.0000 & $\infty$ & $25.0 \%$ & 0.0000 & 177.9 & $20.3 \%$ & 0.0000 & 114.2 \\
\hline Power vs EDF & $93.5 \%$ & 0.0000 & $\infty$ & $42.5 \%$ & 0.0000 & 492.6 & $29.3 \%$ & 0.0000 & 245.6 \\
\hline Expo vs EDF & $94.6 \%$ & 0.0000 & $\infty$ & $43.1 \%$ & 0.0000 & 505.3 & $30.4 \%$ & 0.0000 & 263.6 \\
\hline PCPT vs EDF & $79.5 \%$ & 0.0000 & $\infty$ & $36.1 \%$ & 0.0000 & 366.1 & $24.4 \%$ & 0.0000 & 170.2 \\
\hline CPT vs EDF & $29.4 \%$ & 0.0000 & 246.4 & $7.2 \%$ & 0.0631 & 3.5 & $8.4 \%$ & 0.0048 & 8.0 \\
\hline
\end{tabular}




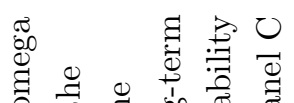

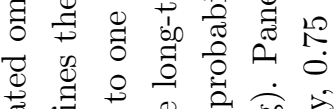

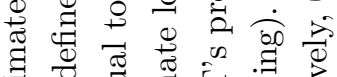

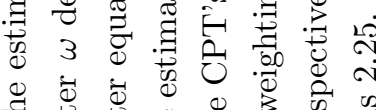

पै

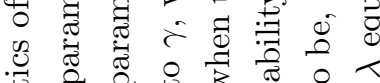

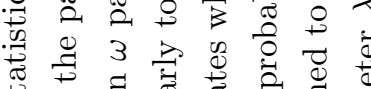

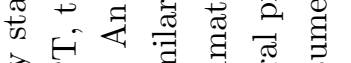

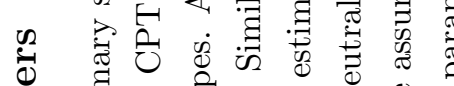

要

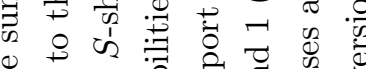

的

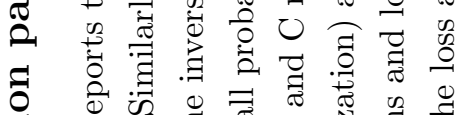

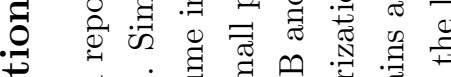

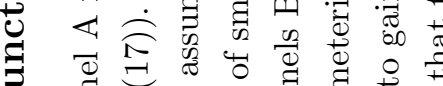

等

然

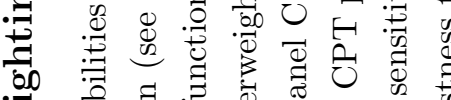

일

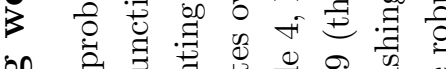

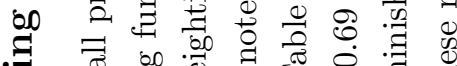

ह

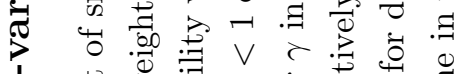

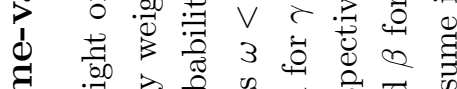

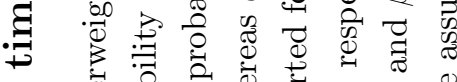

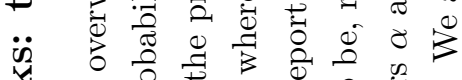

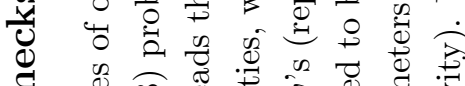

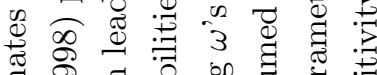

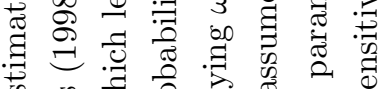

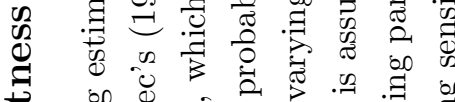

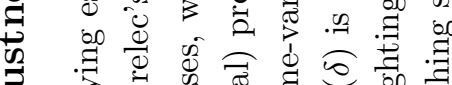
等

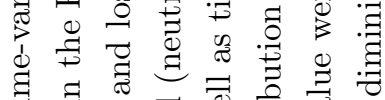

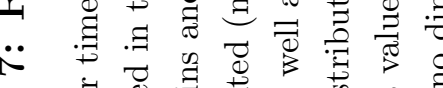

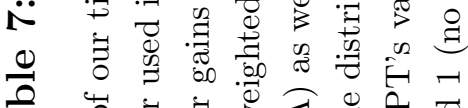

象

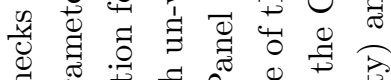

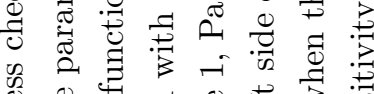

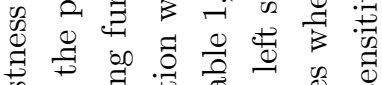

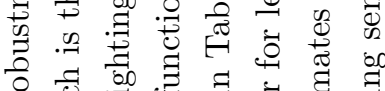

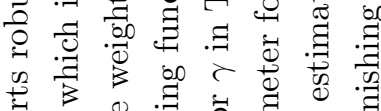

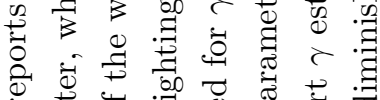

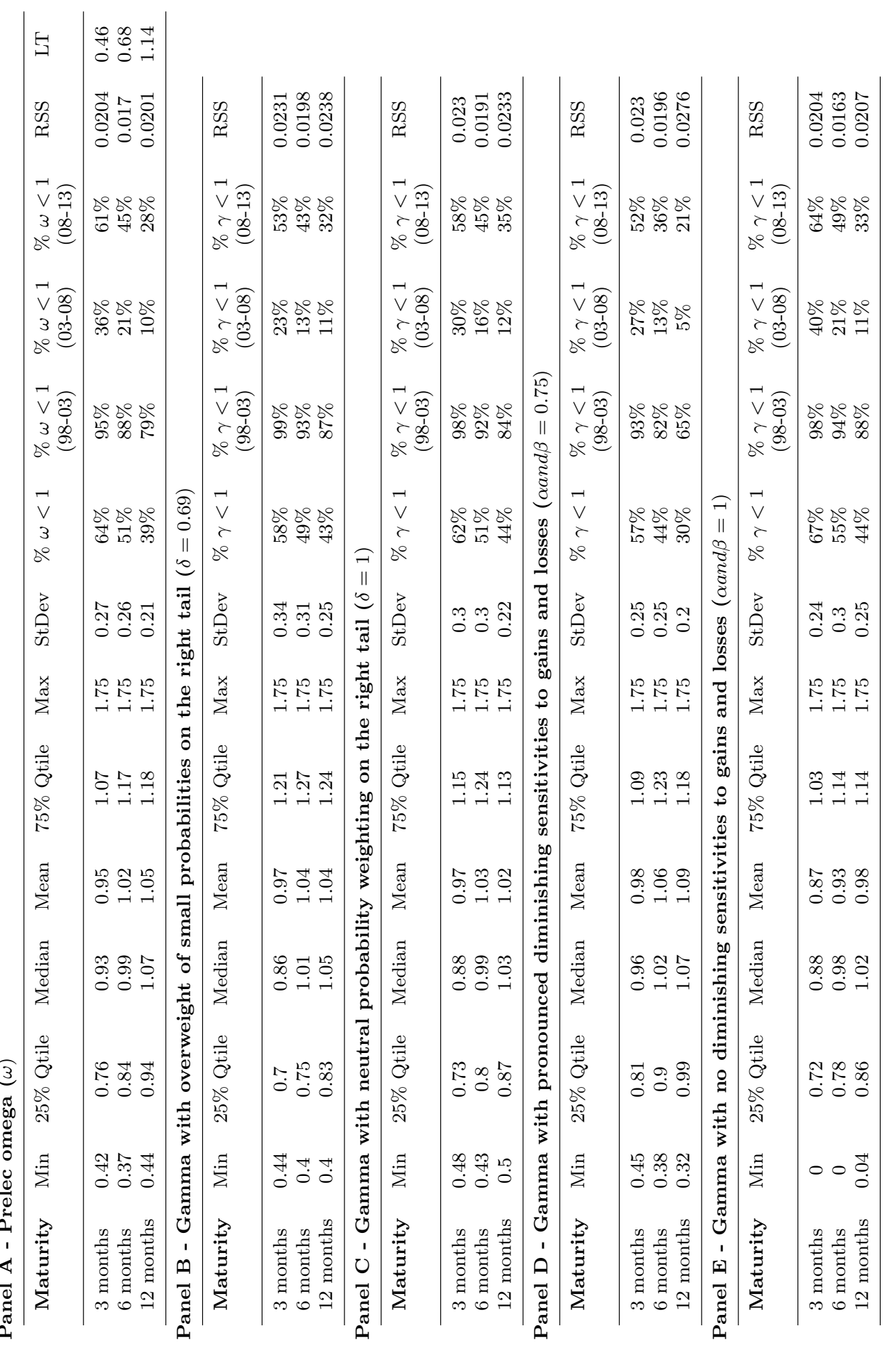

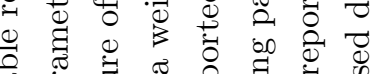

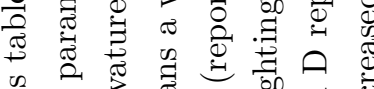

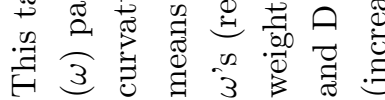




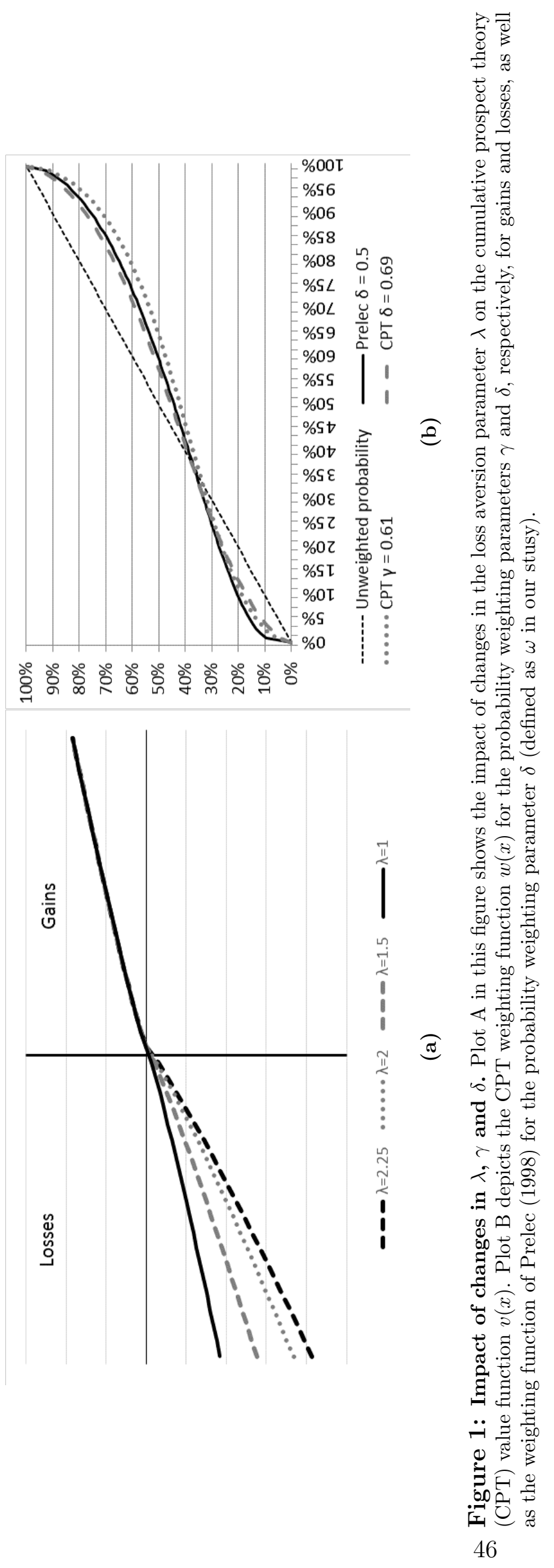




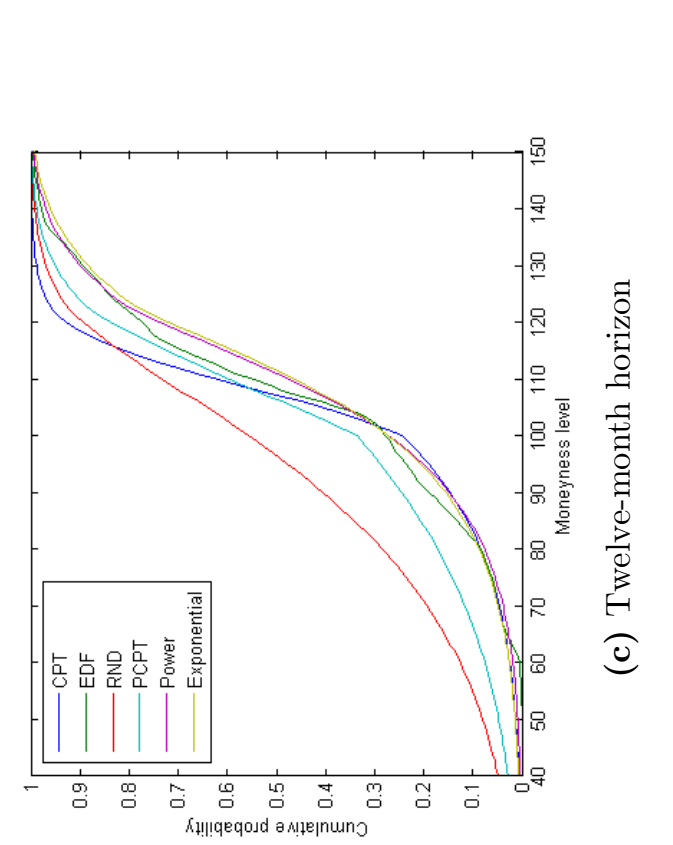

焉

蛋

ठ류

吾造

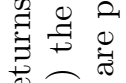

워

兽密兽

实

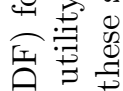

已

궁

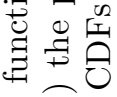

㺃

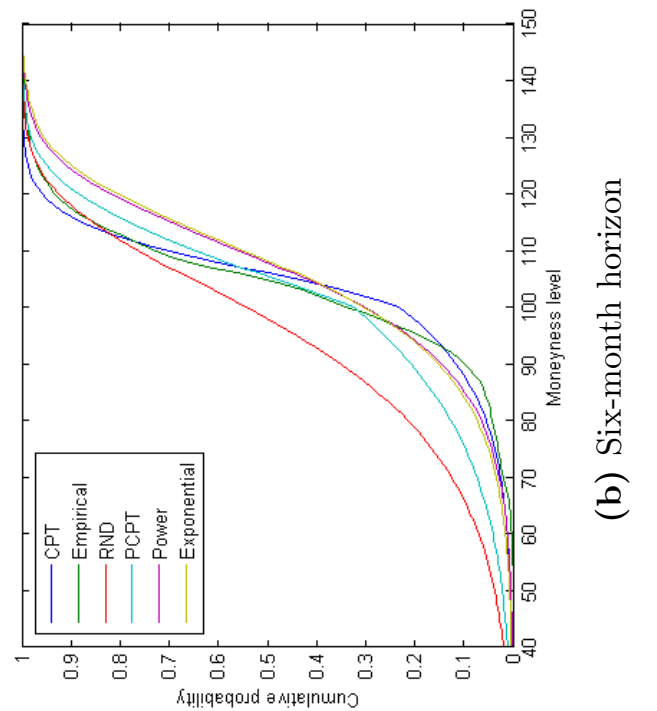

品

莺

列泀

$\pm \dot{0}$

苞它

웡

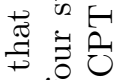

o

뭉

o

ฮี

完宗

की

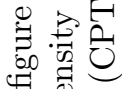

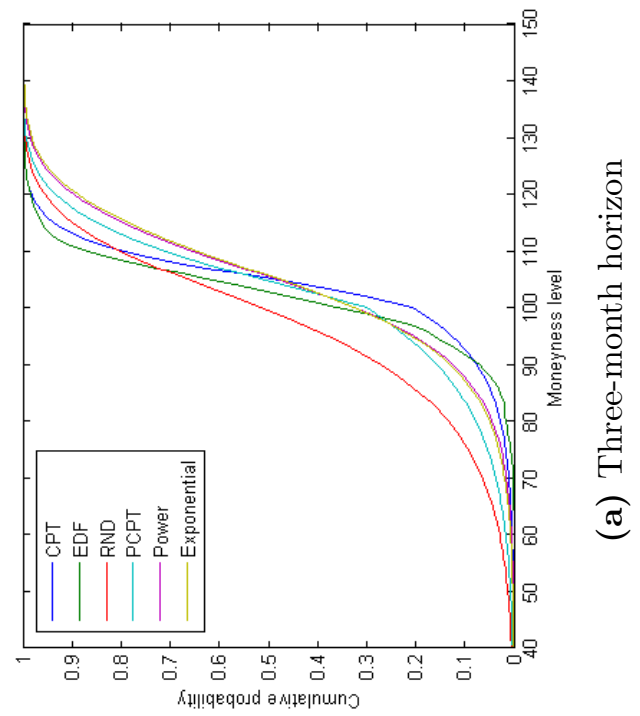

. 8

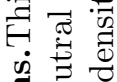

.

章

E

可语

过司通

: 0 ..

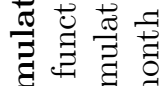

ป

ن

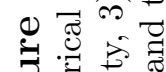




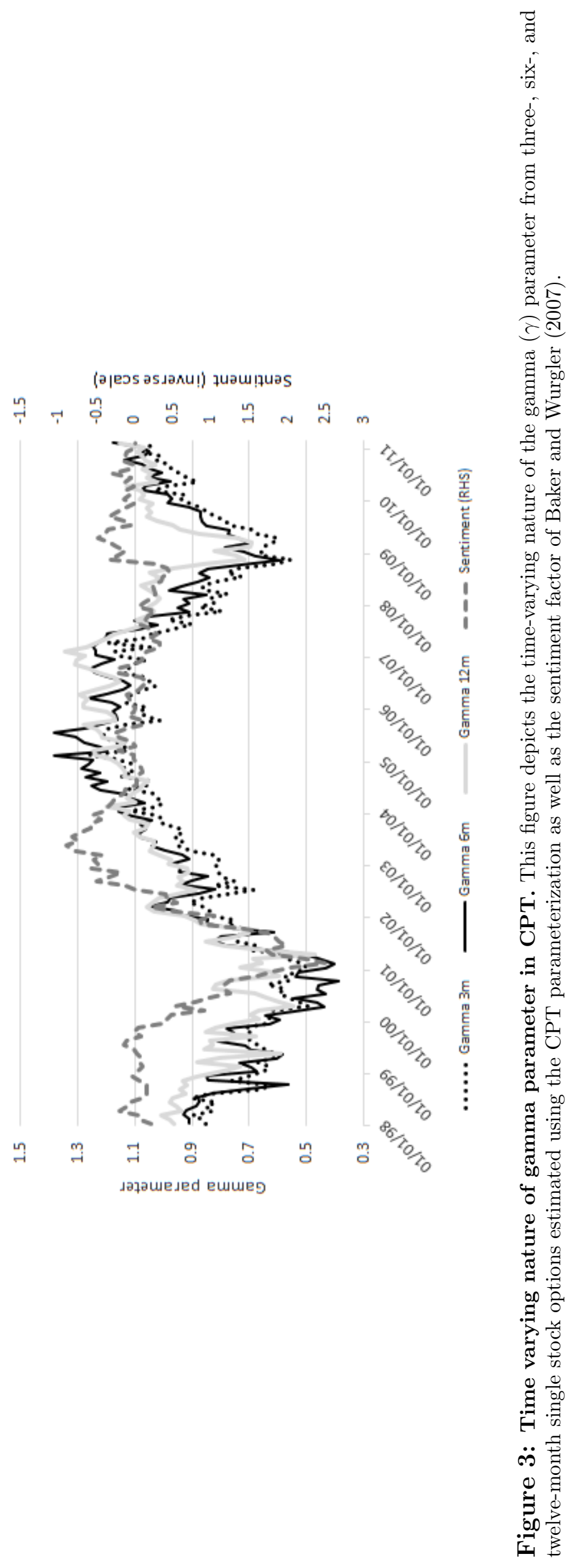




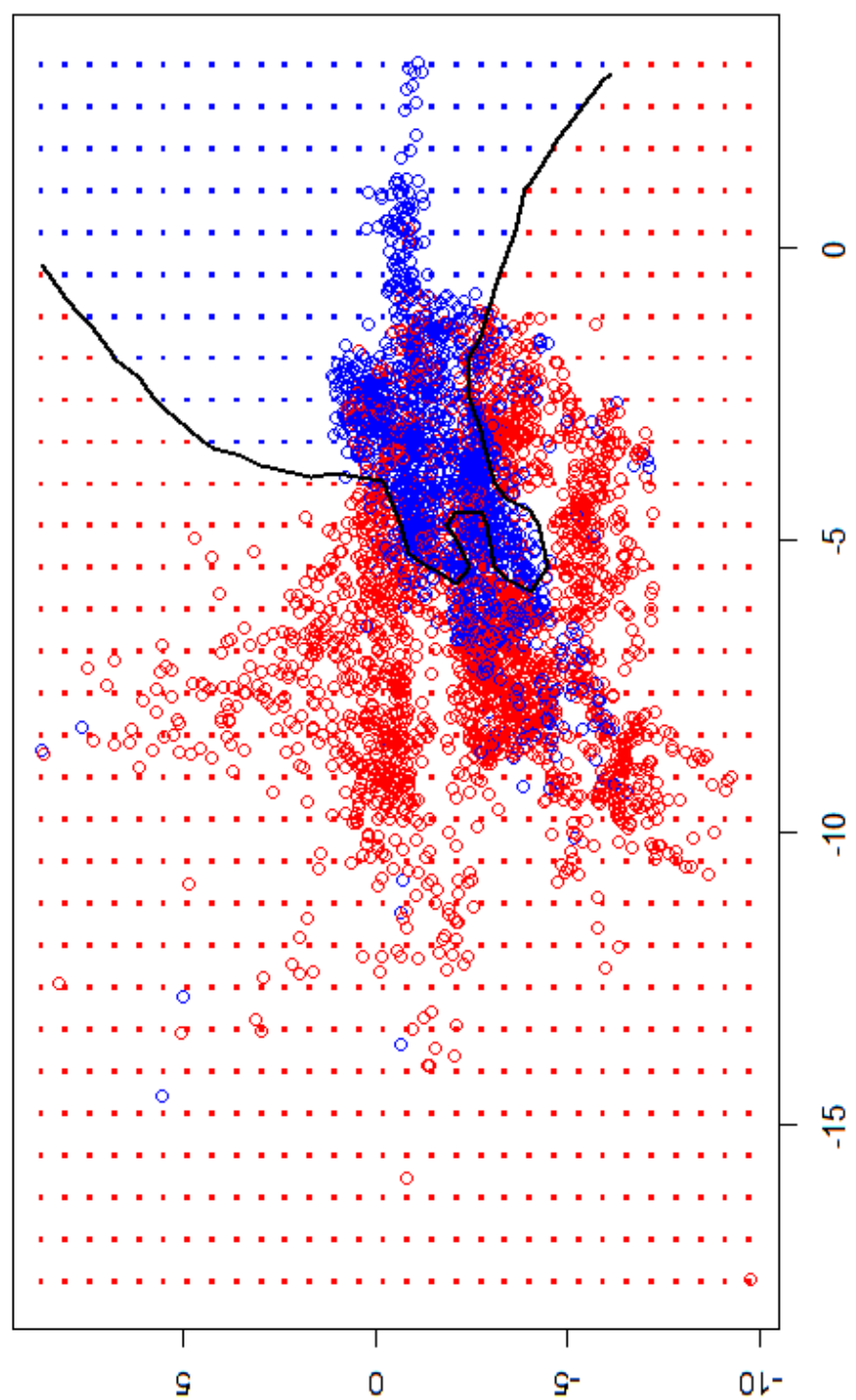

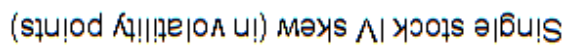

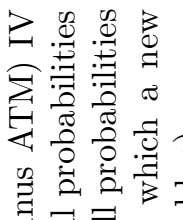

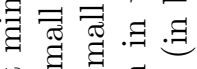

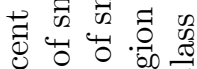

武艺

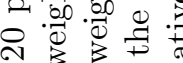

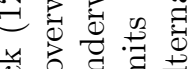

范寻寻

is 0 :

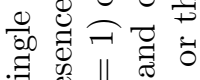

औ气

Ð 00.

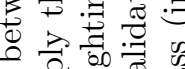

ㄱ. ․ㅣㅇ

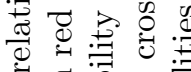

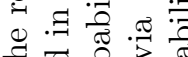

ఫ

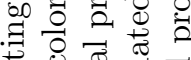

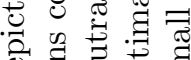

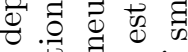

जी

员:

$-100.0$

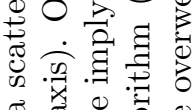

w

秀

क ॠ.

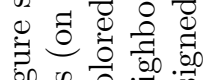

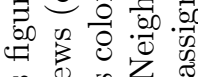

.

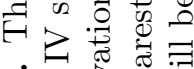

$\dot{m}=\mathbb{0}^{\pi}$

总最点出

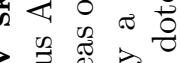

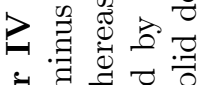

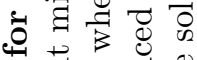

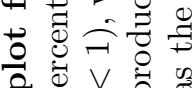

a

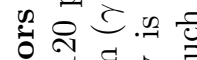
을 귤 क

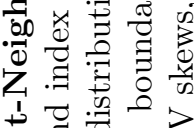

कै

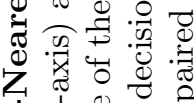

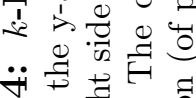

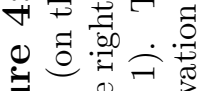

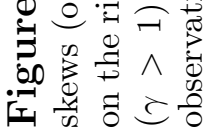

